\title{
The VIMOS-VLT deep survey: the group catalogue ${ }^{\star}$
}

\author{
O. Cucciati ${ }^{1,2}$, C. Marinoni ${ }^{3}$, A. Iovino ${ }^{1}$, S. Bardelli ${ }^{4}$, C. Adami ${ }^{2}$, A. Mazure ${ }^{2}$, M. Scodeggio ${ }^{5}$, D. Maccagni ${ }^{5}$,
} S. Temporin ${ }^{6}$, E. Zucca ${ }^{4}$, G. De Lucia ${ }^{7}$, J. Blaizot ${ }^{8}$, B. Garilli ${ }^{5}$, B. Meneux ${ }^{9}, 10$, G. Zamorani ${ }^{4}$, O. Le Fèvre ${ }^{2}$, A. Cappi ${ }^{4}$, L. Guzzo ${ }^{1}$, D. Bottini ${ }^{5}$, V. Le Brun ${ }^{2}$, L. Tresse ${ }^{2}$, G. Vettolani ${ }^{11}$, A. Zanichelli ${ }^{11}$, S. Arnouts ${ }^{12,2}$, M. Bolzonella ${ }^{4}$, S. Charlot ${ }^{13,14}$, P. Ciliegi $^{4}$, T. Contini ${ }^{15}$, S. Foucaud ${ }^{16}$, P. Franzetti $^{5}$, I. Gavignaud ${ }^{17}$, O. Ilbert ${ }^{2}$, F. Lamareille ${ }^{15}$, H. J. McCracken ${ }^{14,18}$, B. Marano ${ }^{19}$, R. Merighi ${ }^{4}$, S. Paltani ${ }^{20,21}$, R. Pellò ${ }^{15}$, A. Pollo ${ }^{22,23}$, L. Pozzetti ${ }^{4}$, D. Vergani ${ }^{5}$, and E. Pérez-Montero ${ }^{24}$

(Affiliations can be found after the references)

Received 11 February 2009 / Accepted 7 May 2010

\section{ABSTRACT}

Aims. We present a homogeneous and complete catalogue of optical galaxy groups identified in the purely flux-limited $\left(17.5 \leq I_{\mathrm{AB}} \leq 24.0\right)$ VIMOS-VLT deep redshift Survey (VVDS).

Methods. We use mock catalogues extracted from the Millennium Simulation, to correct for potential systematics that might affect the overall distribution as well as the individual properties of the identified systems. Simulated samples allow us to forecast the number and properties of groups that can be potentially found in a survey with VVDS-like selection functions. We use them to correct for the expected incompleteness and, to asses in addition, how well galaxy redshifts trace the line-of-sight velocity dispersion of the underlying mass overdensity. In particular, on these mock catalogues we train the adopted group-finding technique i.e., the Voronoi-Delaunay Method (VDM). The goal is to fine-tune its free parameters, recover in a robust and unbiased way the redshift and velocity dispersion distributions of groups $(n(z)$ and $n(\sigma)$, respectively), and maximize, at the same time, the level of completeness and purity of the group catalogue.

Results. We identify 318 VVDS groups with at least 2 members in the range $0.2 \leq z \leq 1.0$, among which 144 (/30) with at least 3 (/5) members. The sample has an overall completeness of $\sim 60 \%$ and a purity of $\sim 50 \%$. Nearly $45 \%$ of the groups with at least 3 members are still recovered if we run the algorithm with a particular parameter set that maximizes the purity $(\sim 75 \%)$ of the resulting catalogue. We use the group sample to explore the redshift evolution of the fraction $f_{\mathrm{b}}$ of blue galaxies $(U-B \leq 1)$ in the redshift range $0.2 \leq z \leq 1$. We find that the fraction of blue galaxies is significantly lower in groups than in the global population (i.e. in the whole ensemble of galaxies irrespective of their environment). Both of these quantities increase with redshift, the fraction of blue galaxies in groups exhibiting a marginally significant steeper increase. We also investigate the dependence of $f_{\mathrm{b}}$ on group richness: not only we confirm that, at any redshift, the blue fraction decreases in systems with increasing richness, but we find that this result continues to hold towards fainter luminosities.

Key words. galaxies: clusters: general - large-scale structure of Universe - galaxies: high-redshift - galaxies: evolution galaxies: statistics

\section{Introduction}

Galaxy groups and clusters are the largest and most massive gravitationally bound systems in the universe. Because of this, they are very useful cosmological probes. For example, the evolution of either their abundance or baryon fraction provides insights into the value of fundamental cosmological parameters (e.g., Borgani et al. 1999; Newman \& Davis 2002; Allen et al. 2002; Ettori et al. 2003; Zhang et al. 2006; Ettori et al. 2009), their mass and luminosity functions fix the amplitude of the power spectrum on cluster scales (e.g. Rosati et al. 2002; Finoguenov et al. 2010), while their optical mass-to-light ratio allows us to constrain the matter density parameter $\Omega_{\mathrm{m}}$ (e.g. Girardi et al. 2000; Marinoni \& Hudson 2002; Sheldon et al. 2009). Groups and clusters are also ideal laboratories for astrophysical studies. Several interesting physical processes are indeed triggered on scales characterized by these extreme density conditions. Their analysis is crucial in particular to understanding the effects of local environment on galaxy formation and

* Complete Tables 4 and 5 are only available in electronic form at the CDS via anonymous ftp to

cdsarc.u-strasbg.fr (130.79.128.5) or via

http://cdsarc.u-strasbg.fr/viz-bin/qcat?J/A+A/520/A42 evolution (e.g. Oemler 1974; Dressler 1980; Postman \& Geller 1984; Dressler et al. 1997; Garilli et al. 1999; Treu et al. 2003; Poggianti et al. 2006).

\subsection{The detection of galaxy groups and clusters}

A whole arsenal of algorithms allows us to identify and reconstruct galaxy systems. They range from the very first pioneering methods based on visual identification on photometric plates (Abell 1958; Zwicky et al. 1968) to more recent techniques which exploit various physical properties of the systems as a guide for identification. For example, the thermal bremsstrahlung emission from the hot intracluster gas trapped inside the cluster gravitational potential allows us to spot them by means of X-ray band observations. On the opposite side of the spectrum, in the centimetre regime, cluster detection is made possible thanks to the Sunyaev-Zeldovich effect (SZE, Sunyaev \& Zeldovich 1972, 1980). The hot intracluster gas, by means of inverse-Compton scattering the photons of the cosmic microwave background (CMB), leaves a characteristic imprint on the CMB spectrum, which can be exploited as a useful signature for identification. A cluster potential well can also be detected 
from the strong gravitational lensing or the cosmic shear induced by weak gravitational lensing (Kneib et al. 2003; Gavazzi et al. 2009; Limousin et al. 2009; Richard et al. 2010; Limousin et al. 2010; Morandi et al. 2010). Clusters identification can be based also on the properties of the member galaxies. Cluster cores are found to host typically red galaxies, among which there are the brightest cluster galaxies (BCG). Thus, a cluster center can be identified as a $R A-d e c$ concentration of galaxies with typical red colours (see for example the Red-Sequence Cluster Survey, Gladders \& Yee 2000, the first cluster survey based on this method), in some cases also adding the constraint of a high luminosity (e.g. the $\max B C G$ method, Hansen et al. 2005; Koester et al. 2007).

An orthogonal approach, based on geometrical algorithms, consists of identifying systems from the 3D spatial distribution properties of their members. These algorithms vary from the earlier hierarchical method (Materne 1978; Tully 1980) and the widely used "friend of friend" (FOF) method (Huchra \& Geller 1982), to the 3D adaptive matched filter method (Kepner et al. 1999), the "C4" method (Miller et al. 2005), and the VoronoiDelaunay Method (VDM, Marinoni et al. 2002). Finally, groupfinding algorithms have been developed that use information extracted from photometric redshifts (e.g. Adami et al. 2005; Mazure et al. 2007).

The availability of several identification protocols is not only useful for confirming cluster detections by an a posteriori crosscorrelation of various independent catalogues, but it is also crucial for anticipating systematics that might affect individual detection techniques. For example, it was shown by the first joint X-ray/optical survey (Donahue et al. 2002) that only 20\% of optically selected clusters appeared to be identifiable in X-rays, while $\sim 60 \%$ of the X-ray clusters were included in the optical sample. Understanding the possible selection effects hidden behind the different survey strategies is crucial to explain the small size of the overlap between the two different cluster catalogues (see for example Ledlow et al. 2003; Gilbank et al. 2004). Moreover, using the RASS-SDSS galaxy cluster catalogue, Popesso et al. (2004) show that a distinct class of "X-ray underluminous Abell clusters" does exist, with an X-ray luminosity $L_{X}$ that is one order of magnitude fainter than that expected for their mass according to the typical $L_{X}$-mass relation (Popesso et al. 2007a). This supports the concern of Donahue et al. (2002) about the possible existence of biases in catalogues selected in different wavebands.

A major challenge that we face is to extend cluster searches to high redshift. Most of the methods described above are affected by major problems when applied to this regime. Both the X-ray apparent surface brightness and the gravitational lensing cross-section of clusters decrease very rapidly with redshift. As a consequence, only very massive clusters can be detected at high $z$. In contrast, the SZE detection efficiency does not depend on redshift, but large SZ survey are yet to be completed. When detecting clusters using the spatial distribution of members, we emphasize the difference between photometric and spectroscopic galaxy data sets. Several methods have been proposed to detect clusters with photometric data, mainly exploiting galaxy colours in different bands. On the one side, this method has been successfully used both for surveys (see for example the above-mentioned Red-Sequence Cluster Survey, Gladders \& Yee 2000) and single detections (e.g. Andreon et al. 2009). On the other hand, the selection of red galaxies, however, is biased towards older structures, where galaxies have lived enough time to be affected by the physical processes typical of the group environment (see for example the discussion in
Gerke et al. 2007). Moreover, the depth required in photometric surveys to identify high- $z$ groups and clusters increases the number of foreground and background contaminant galaxies, as the object surface number density is increased by the faint flux limit. This limits the effectiveness of 2D identifications at high- $z$. Knowledge of the third dimension is thus imperative if we wish to mitigate projection effects in an efficient way. Nonetheless, the uncertainty in the line-of-sight (l.o.s.) position of galaxies may be a concern when it is larger (or even far larger) than the typical velocity dispersion of group galaxies, as is typically the case for photometric redshifts.

\subsection{This work and existing group and cluster samples}

To date, many local, optically selected group catalogues are available in literature. A review can be found in Eke et al. (2004), where one of the largest catalogue of galaxy groups detected in redshift space from the Two Degree Field Galaxy Redshift Survey (2dFGRS) is presented. In addition, several group catalogues have been extracted from the Sloan Digital Sky Survey data (e.g. Miller et al. 2005; Berlind et al. 2006; Weinmann et al. 2006). Systematic searches of groups in redshift space have also been undertaken at intermediate redshift (e.g. within the CNOC2 survey, up to redshift $z=0.55$, Carlberg et al. 2001). The compilation of optically selected and complete samples of groups up to $z \sim 1$ and beyond has become possible only recently thanks to the completion of large and deep spectroscopic surveys, such as the DEEP2 Galaxy Redshift Survey (Davis et al. 2003), the VIMOS-VLT deep survey (Le Fèvre et al. 2005), and the zCOSMOS survey (Lilly et al. 2007, 2009).

Gerke et al. (2005) present the first DEEP2 group catalogue, which contains 899 groups with two or more members identified in the redshift range $0.7 \leq z \leq 1.4$ with the VDM method. The DEEP2 sample reaches a limiting magnitude of $R_{\mathrm{AB}}=24.1$, and its galaxies were pre-selected in colour before being targeted for spectroscopic observations, to reduce the number of galaxies at $z \lesssim 0.7$. The first zCOSMOS group catalogue (Knobel et al. 2009) comprises $\sim 800$ groups with at least 2 members, covering the redshift range $0.1 \leq z \leq 1.0$. The parent galaxy sample is purely flux limited $\left(15 \leq I_{\mathrm{AB}} \leq 22.5\right)$, and groups were detected with the FOF method, combined with the VDM.

In this work, we use the VIMOS-VLT deep survey (VVDS, Le Fèvre et al. 2005) to compile a homogeneous opticallyselected group catalogue in the redshift range $0.2<z<1.0$. We executed the VDM code for a sample containing more than 6000 flux-limited galaxies $\left(17.5 \leq I_{\mathrm{AB}} \leq 24.0\right)$ for which reliable spectroscopic redshifts had been measured. Particular care was taken to optimally tune the parameters of the group-finding algorithm using VVDS-like mock catalogues. The selection function of the sample, which compensates only for the flux limitedness of the survey, is simple and mostly insensitive to possibly uncontrolled biases such as those affecting colour-selected samples. Moreover, the magnitude depth of the VVDS allows us to select a galaxy population that is fainter in luminosity than that currently probed by other flux-limited surveys of the deep universe.

The paper is organized as follows. In Sect. 2, the data sample and the mock catalogues are described. The reliability of the virial 1.o.s. velocity dispersion estimated using galaxies is discussed in Sect. 3. In Sect. 4, we review the basics of the VDM group-finding algorithm, while the strategy followed to ascertain an optimal set of parameters is presented in Sect. 5. In Sect. 6, we describe the properties of the VVDS group catalogue. The redshift evolution of the $U-B$ colour of group galaxies is analyzed in Sect. 7. Conclusions are drawn in Sect. 8. 
We frame our analysis in the context of a $\Lambda$ Cold Dark Matter model $(\Lambda \mathrm{CDM})$ specified by the parameters $\Omega_{\mathrm{m}}=0.3, \Omega_{\Lambda}=$ 0.7 , and $H_{0}=70 \mathrm{~km} \mathrm{~s}^{-1} \mathrm{Mpc}^{-1}$. Magnitudes are expressed in the $\mathrm{AB}$ system.

\section{Data sample and mock catalogues}

\subsection{The VVDS-02h sample}

The VIMOS-VLT deep survey (VVDS) is a large spectroscopic survey whose primary aim is to study galaxy evolution and large-scale structure formation. The survey strategy and goals are described in detail by Le Fèvre et al. (2005). The VVDS is complemented by ancillary deep photometric data that have been collected at the CFHT telescope (BVRI, Le Fèvre et al. 2004; McCracken et al. 2003), at the NTT telescope (JK, Iovino et al. 2005; Temporin et al. 2008), and at the MPI telescope ( $U$, Radovich et al. 2004). In addition, $u^{*}, g^{\prime}, r^{\prime}, i^{\prime}$, and $z^{\prime}-$ band data are available as part of the CFHT Legacy Survey. The full suite of spectroscopic and photometric data provides a superb database to address across a wide redshift range many open questions of modern observational cosmology.

In this paper, we use data collected for the VVDS-0226-04 deep field (from now on "VVDS-02h field"), for which the spectroscopic observations targeted objects in the magnitude range $17.5 \leq I_{\mathrm{AB}} \leq 24.0$. In this range, the parent photometric sample is complete and free of surface brightness selection effects (McCracken et al. 2003), resulting in a deep and purely fluxlimited spectroscopic sample. Spectroscopic observations (the so-called "first epoch" data) in the VVDS-02h field were carried out at the ESO-VLT with the VIsible Multi-Object Spectrograph (VIMOS), a 4-channel imaging spectrograph, each channel (a quadrant) covering $\sim 7 \times 8 \mathrm{arcmin}^{2}$ for a total field of view (a pointing) of $\sim 218 \operatorname{arcmin}^{2}$. The observations used 1 arcsec wide slits and the LRRed grism, covering the spectral range $5500 \AA<\lambda<9400 \AA$. The resulting effective spectral resolution is $R \sim 227$, while the rms accuracy of the redshift measurements is $\sim 275 \mathrm{~km} \mathrm{~s}^{-1}$ (Le Fèvre et al. 2005).

The VVDS-02h field covers a total sky area of $0.7 \times 0.7 \mathrm{deg}^{2}$, targeted by 1,2 , or 4 spectrograph passes. This strategy produces an uneven target sampling rate as shown in Fig. 1. The multiplepass strategy ensures that there is no serious undersampling of the denser regions, at least in the $\sim 80 \%$ of the field covered by two or more spectrograph passes. We note that some quadrants were discarded because of their poor quality and not all the regions of the field covered by the same number of passes have the same sampling rate. On average, spectra were obtained for a total of $22.8 \%$ of the parent photometric catalogue. Owing to the low signal-to-noise ratio and/or to the absence of useful spectral features, redshifts are available for only $\sim 80 \%$ of these targeted objects, giving an overall sampling rate of $\sim 18 \%$ ( $\sim 33 \%$ considering only the area covered by 4 passes).

The VVDS-02h field first epoch sample probes a comoving volume (up to $z=1.5$ ) of nearly $1.5 \times 10^{6} h^{-3} \mathrm{Mpc}^{3}$ in a standard $\Lambda \mathrm{CDM}$ cosmology. This volume has transversal dimensions $\sim 37 \times 37 h^{-1} \mathrm{Mpc}$ at $z=1.5$ and extends over $3060 h^{-1} \mathrm{Mpc}$ in the radial direction.

The collected sample contains 6615 galaxies and AGNs with secure redshifts, i.e., redshift determined with a quality flag $=2$, $3,4$, and 9 (6058 with $0.2 \leq z \leq 1.5)$. We refer the reader to Le Fèvre et al. (2005) for further details about redshift quality flags. By comparing spectroscopic redshifts of objects observed twice in independent observations, we note only that redshifts with flag $=2(/ 3 / 4)$ are correctly estimated with a likelihood of $81(/ 97 />99) \%$. We assigned a flag $=9$ to a spectrum with only

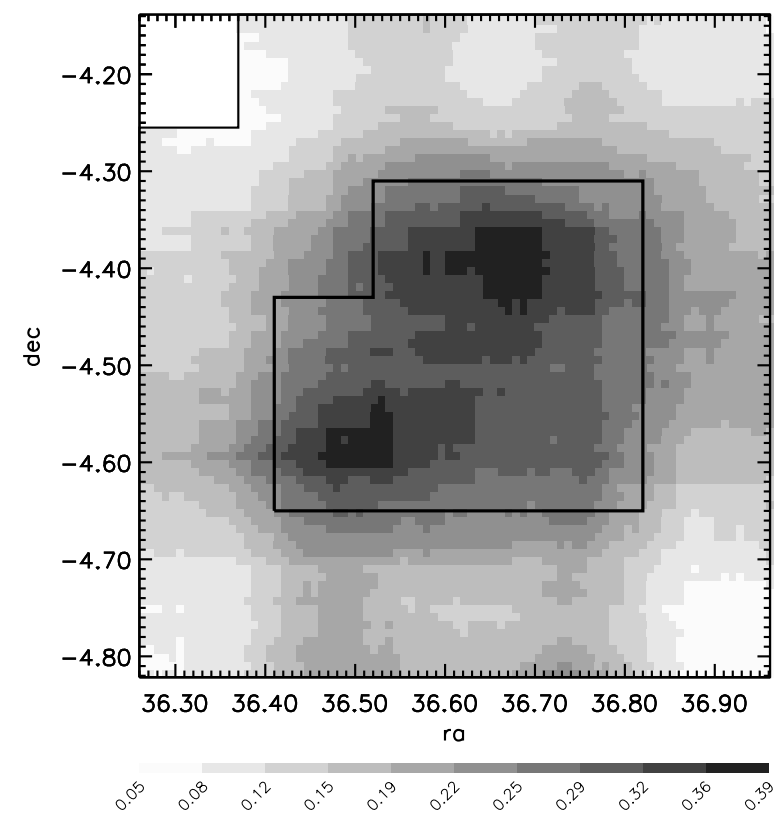

Fig. 1. Uneven spectrograph coverage in VVDS-02h field. The greyscale from the lightest to the darkest grey indicates the sampling rate, with corresponding values shown in the label. The grid used for the colour-code had steps of 30" in right ascension and declination, and for each grid position we used squares of size $7^{\prime}$ to estimate the sampling rate. The central area covered by 4 VIMOS passes is highlighted by a solid line.

a single secure spectral feature in emission. Given the spectral range covered by observations and the flux limits of the survey, this emission line is typically [OII] $3727 \AA$ or $\mathrm{H} \alpha$ (in very rare cases $\operatorname{Ly} \alpha$ ). Thus flag $=9$ redshifts have a probability of being correct of $\sim 50 \%$, being based on the choice between the two most probable emission lines. We double-check the robustness of the likelihood assigned to flag $=2$ and flag $=9$ objects, by comparing their spectroscopic and photometric redshifts. Photometric redshifts were computed as described in Ilbert et al. (2006), but using the more recent T0005 release of CFHTLS data $\left(u^{*}, g^{\prime}, r^{\prime}, i^{\prime}, z^{\prime}\right.$ filters) and the latest data available from WIRCAM ( $J, H$ and $K$ filters, Bielby et al. in prep.). According to the comparison of the two types of redshift, flag $=2(/ 9)$ redshifts are correctly inferred with a likelihood of 78(/59)\%, a figure that is in good agreement with the independent determination discussed above.

We note also that the conclusions of our work are unaffected by our including or not flag $=9$ low quality redshifts in our analysis. As a matter of fact, these objects constitute a small fraction $(<3 \%)$ of the whole sample. Moreover, the effect of possible biases induced by incorrect redshift estimates is weakened by the existence of the galaxy correlation on small scales: if a galaxy with flag $=2$ is located close (on the sky) to other galaxies with similar (but more secure) redshifts, the likelihood that it shares the same redshift actually increases with respect to the probability determined on the basis of our analysis.

\subsection{Mock catalogues}

We made extensive use of mock catalogues, both to test the potential for group searches of the VVDS-02h field data and to tune the parameters of the group-finding algorithm for optimal detection. 
Before introducing any particular group-finding algorithm, one needs to test the limits to group reconstruction imposed by the specific characteristics of the VVDS survey design. Using mock catalogues that mimic the VVDS-02h field, we were able to explore which groups are lost irretrievably due to the survey sparse galaxy sampling. Furthermore, we were able to assess how our measurement of the l.o.s. velocity dispersion of group galaxies is degraded by both the sampling rate and the non-negligible VVDS redshift measurement error. After exploring these limits, we then moved to test and optimize the groupfinding algorithm, within the ranges in redshift and velocity dispersion where we found that VVDS-02h data allow a reliable group reconstruction.

Mock catalogues were obtained by applying the semianalytic prescriptions of De Lucia \& Blaizot (2007) to the dark matter halo merging trees extracted from the Millennium Simulation $^{1}$ (Springel et al. 2005). The simulation contains $N=$ $2160^{3}$ particles of mass $8.6 \times 10^{8} h^{-1} M_{\odot}$ within a comoving box of size $500 h^{-1} \mathrm{Mpc}$ on a side. The cosmological model is a $\Lambda C D M$ model with $\Omega_{\mathrm{m}}=0.25, \Omega_{\mathrm{b}}=0.045, h=0.73$, $\Omega_{\Lambda}=0.75, n=1$, and $\sigma_{8}=0.9$. The positions and velocities of all simulated particles were stored at 63 snapshots, spaced approximately logarithmically from $z=20$ to the present day. Dark matter halos were identified using a standard friends-of-friends (FOF) algorithm with a linking length of 0.2 in units of the mean particle separation.

In this simulation, group galaxies were assumed to be those in the same FOF halo, identified with a unique ID. For each simulated group, a wealth of physical information are available: galaxy membership, virial mass (computed directly using the simulated particles), virial radius, and virial velocity dispersion (both inferred from the virial mass, by means of scaling laws and the virial theorem). The virial mass is evaluated within the radius where the halo has an overdensity 200 times the critical density of the simulation.

We note that the model used to construct light-cones from the Millennium Simulation has been shown to be quite successful in reproducing several basic properties of our real data set. The most important are the average redshift distribution $n(z)$ (Meneux et al. 2008) and the global luminosity function (LF) (Zucca et al., in prep.), which are in good agreement with the real VVDS-02h $n(z)$ and LF, the only exception being a slight excess of galaxies in the $n(z)$ mock samples for $z<0.5$. This small difference in $n(z)$ does not affect the completeness and purity values (see Sect. 5.1) of our group catalogue, as we specifically tested using separately the mocks with the most similar and the most different $n(z)$. Moreover, Meneux et al. (2008) demonstrated that the galaxy clustering in the Millennium Simulation light cones is consistent with that measured using the VVDS-02h sample.

From the database compiled from the Millennium Simulation (Lemson \& Virgo Consortium 2006), we selected $10(1 \times$ 1) $\mathrm{deg}^{2}$ independent Millennium light cones (generated with the code MoMaF, Blaizot et al. 2005), from which we extracted several kinds of mocks, according to our purposes. First of all, we extracted $(1 \times 1)$ deg $^{2}$ flux-limited samples with the same flux limits as VVDS-02h sample $\left(17.5 \leq I_{\mathrm{AB}} \leq 24\right)$. These catalogues each have a $100 \%$ sampling rate, and no redshift measurement error has been added. We called these catalogues $M(100,0)$, the first number in brackets indicating the sampling rate and the second the redshift error. We then randomly depopulated these catalogues to obtain subsets with $33 \%, 17 \%$,

\footnotetext{
${ }^{1}$ http://www.mpa-garching.mpg.de/galform/virgo/ millennium/
}

and $10 \%$ sampling rates, mimicking roughly the sampling rate of the 4 pass, 2 pass and 1 pass areas of the VVDS-02h field. These catalogues are called $M(33,0), M(17,0)$, and $M(10,0)$ respectively. With these mock catalogues and taking advantage of the known group membership, we were able to determine the extent to which a group catalogue is depopulated when the sampling rate is decreased to values typical of those of VVDS-02h field.

As an additional step, we added redshift measurement errors to the $33 \%$ sampling rate mocks, randomly chosen from a Gaussian distribution centered on 0 with $\sigma=275 \mathrm{~km} \mathrm{~s}^{-1}$. In this way, we took account of the mean redshift measurement error in our true data. We called these mock catalogues $M(33,275)$. With these mock catalogues, we were able to test how well we can determine group virial velocity dispersion when the survey has flux limits, sampling rate, and redshift measurement errors mimicking those of the 4 pass areas of the VVDS-02h field.

As a last step, we needed mock catalogues to test how effective the group-finding algorithm that we adopted is in identifying groups surviving in a sample such as VVDS-02h. To test the efficiency of our algorithm, we used 20 "VVDS-like" mocks extracted from the Millennium Simulation. These mocks have the same flux limits, geometry, uneven sampling rate, and redshift error measurement as the VVDS-02h sample (see Pollo et al. 2005; and Meneux et al. 2008, for the preparation of these mocks). Subtler effects, such as those introduced by a slit positioning bias, were also included, because the same slit positioning tool used for the VVDS-02h sample was used, with the same optimization criteria, to generate the VVDS-like mocks. The areas masked in the true photometric catalogue because of either bright stars and a beam of scattered light were also masked in the VVDS-like mocks.

For the sake of clarity, we emphasize that whenever we refer to the "FOF" or "simulated" groups in all the above-mentioned mock catalogues, we mean the sets of galaxies within the same original FOF halo provided by the simulation itself, before any depopulating process: we never executed any FOF algorithm on mocks after extracting $M(100,0), M(33,0), M(17,0), M(10,0)$, $M(33,275)$, and "VVDS-like" mocks from simulations.

\section{Preliminary tests}

\subsection{Testing the effects of VVDS survey strategy on groups}

We explored how closely a group catalogue extracted from a VVDS-like survey represents the group population of an ideal survey that is purely flux-limited. In a real flux-limited galaxy survey with a sampling rate lower than $100 \%$, most groups have a smaller number of members and some even go undetected. We wish to assess the fraction of groups that "survive" as such (i.e. with at least 2 members) in a survey with a sampling rate similar to that of VVDS-02h. To identify groups, in both the full flux-limited and the various "observed" catalogues, we used at this phase the identification number of FOF groups in the Millennium database. In other words, we consider only the limitations introduced by the survey strategy, neglecting for the moment additional complications introduced by the incompleteness/failures of the specific group finding algorithm that we used.

In Fig. 2, we plot the fraction of groups in mock catalogues that are flux-limited at $17.5 \leq I_{\mathrm{AB}} \leq 24.0$, surviving after applying a sampling rate corresponding to that of the $1 / 2 / 4$ pass regions (i.e. $10 \%, 17 \%$ and $33 \%$, respectively) as indicated by different lines. In practice, we plot the ratio of the number of 


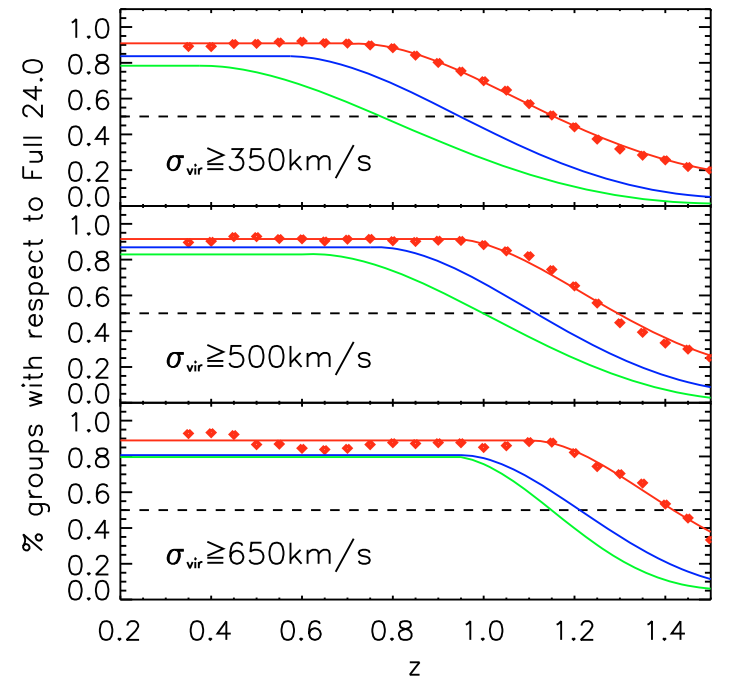

Fig. 2. The fraction, as a function of redshift, of "surviving" groups when the sampling rate is decreased from that of a purely flux-limited simulated sample $17.5 \leq I_{\mathrm{AB}} \leq 24.0$ (i.e. with $100 \%$ sampling rate) to $\sim 33 \%$ ( 4 passes area, red line), $\sim 17 \%$ ( 2 passes, blue line) and $\sim 10 \%$ ( 1 pass area, green line). The fraction was computed in nonindependent running redshift bins of $\Delta z=0.3$ : continuous lines represent fits to all the bins, while for reference the fractions corresponding to the $M(33,0)$ catalogues are also shown for each redshift bin as red diamonds. Different panels show different cuts in $\sigma_{\text {vir }}$, as quoted in each panel. The horizontal dashed line at a fraction value equal to $50 \%$ is for reference.

groups in $M(10,0), M(17,0)$ and $M(33,0)$ catalogues to the number of groups in $M(100,0)$ catalogues. This ratio was computed in non-independent running redshift bins of $\Delta z=0.3$ : continuous lines are fits along all the bins, while for reference the ratios corresponding to the $M(33,0)$ catalogues are also shown for each redshift bin as red diamonds. We note that the number of groups with $\sigma_{\text {vir }} \geq 650 \mathrm{~km} \mathrm{~s}^{-1}$ is quite low, mainly because of the small field of view, thus the fraction of survived groups at $z \leq 0.8$ fluctuates about a mean value that we use to fit a straight line. These fluctuations, however, are as high as $10 \%$ only in the worst cases. This is also true for the $M(10,0)$ and $M(17,0)$ catalogues, for which we do not plot single points to avoid crowding the figure. The horizontal dashed line at a fraction value equal to $50 \%$ is shown for reference. The three panels correspond to different cuts in the virial l.o.s. velocity dispersion $\left(\sigma_{\text {vir }}\right)$ quoted in the mocks, as indicated by the label (from now onwards all velocity dispersions quoted are 1.o.s. velocity dispersions).

Figure 2 shows that in 2 and 4 pass areas we can recover the majority $(\geq 50 \%)$ of groups to $\sigma_{\mathrm{vir}} \sim 350 \mathrm{~km} \mathrm{~s}^{-1}$ in the full redshift range below $z=1.0$. Going to higher values of $\sigma_{\text {vir }}$ obviously allows us to extend the redshift range. This lower limit to $\sigma_{\text {vir }}$ agrees with that implied by the non-negligible redshift measurement error of VVDS survey. As we see in the next paragraph, our measurements of velocity dispersion for groups with $\sigma_{\text {vir }} \leq 350 \mathrm{~km} \mathrm{~s}^{-1}$ are quite unreliable.

\subsection{Estimating group virial l.o.s. velocity dispersion}

A robust determination of the 1.o.s. velocity dispersion of galaxies in group is essential to obtaining a reliable group mass. When group members are sparsely sampled, as in the case of
VVDS-02h data, the "gapper method", originally suggested by Beers et al. (1990), has proved to be the most robust velocity dispersion estimator (see also Girardi et al. 1993). This method measures velocity dispersion by analyzing the velocity gaps in the given velocity distribution of galaxies, using the formula

$\sigma_{\mathrm{G}}=\frac{\sqrt{(} \pi)}{N(N-1)} \sum_{i=1}^{N-1} i(N-i)\left(v_{i+1}-v_{i}\right)$,

where the l.o.s. velocities $v_{i}$ are sorted into ascending order. Beers et al. (1990) show in their Table II that this method reliably estimates the velocity dispersion with an efficiency $>90 \%$ for groups with $\sim 5-10$ elements, thanks to its robustness in recovering the dispersion in a distribution even in the more general case of a contaminated Gaussian distribution. We emphasize that this range of group members is well suited to the study we present in this work. On the one hand, we consider the velocity dispersion reliably measurable only for groups with at least 5 members, and on the other hand the vast majority of groups surviving in "VVDS-like" mocks have $\leq 10$ members.

Hereafter, when discussing "measured" velocity dispersions $\left(\sigma_{\text {meas }}\right)$ we refer to velocity dispersions obtained by applying the gapper method to the members of the given group. Of course, we corrected this velocity dispersion by taking into account the scaling between redshift and velocity, using

$\sigma_{\text {meas }}=\frac{\sigma_{\mathrm{G}}}{1+z}$

where $z$ is the redshift of the group.

We wish to test whether our measurement of the 1.o.s velocity dispersion $\sigma_{\text {meas }}$ is a reliable estimate of the virial velocity dispersion $\sigma_{\text {vir }}$ (as listed in the mock catalogues). For this comparison, we used $M(100,0), M(33,0)$, and $M(33,275)$. We called the value of $\sigma_{\text {meas }}$ measured for these three kinds of catalogues $\sigma(100,0), \sigma(33,0)$, and $\sigma(33,275)$, respectively. In the case of a non-zero redshift measurement error, such as in $M(33,275)$ mock catalogues, we took the error itself into account when computing $\sigma_{\text {meas }}$, by subtracting the error $\left(v_{\text {err }}\right)$ in quadrature as

$\sigma(33,275)^{2}=\frac{\max \left[0, \sigma(33,275)_{\mathrm{G}}^{2}-v_{\mathrm{err}}^{2}\right]}{(1+z)^{2}}$,

where $\sigma(33,275)_{\mathrm{G}}$ is the velocity dispersion measured in $M(33,275)$ mocks with Eq. (1), $v_{\text {err }}=275 \mathrm{~km} \mathrm{~s}^{-1}$, and $z$ is the redshift of the group. When $\sigma(33,275)=0$, we considered the velocity to be unmeasurable given the redshift error.

Figure 3 shows the comparison of $\sigma_{\text {vir }}$ with $\sigma(100,0)$, $\sigma(33,0)$, and $\sigma(33,275)$, respectively in the first, second, and third columns. The first row is for the redshift bin $0.2<z<0.6$, and the second for $0.6<z<1.0$. In each plot, the upper panel shows isodensity contours in the plane $\sigma_{\text {meas }}$ versus $\sigma_{\text {vir }}$ for groups with at least 5 members. Blue triangles are the median (on $x$ axis) and mean (on $y$ axis) values of single points grouped in bins of $\sigma_{\mathrm{vir}}$, with vertical error bars being the rms of mean values. As a reference, purple squares are the same as triangles but for groups with at least 8 members. The lower panel in each plot shows the systematic offset of the relation in the upper panel; the offset is expressed as a percentage error (with its rms) computed to be

err. $\%=\frac{\left[\sigma_{\text {meas }}-\sigma_{\mathrm{vir}}\right]}{\sigma_{\mathrm{vir}}} \times 100$ 

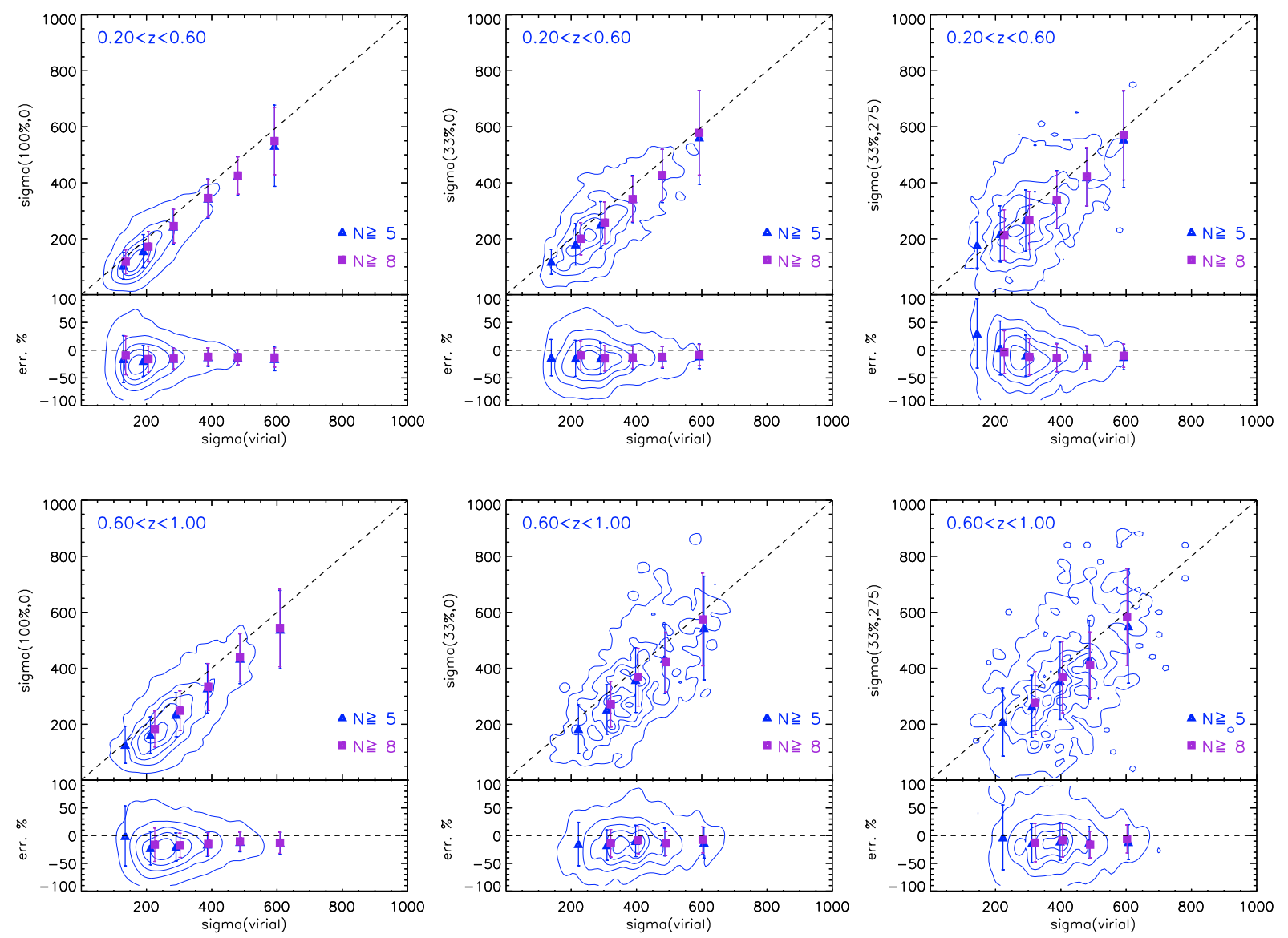

Fig. 3. Comparison of 1.o.s. velocity dispersion $\sigma_{\text {meas }}$ computed in $M(100,0), M(33,0)$ and $M(33,275)$ mocks $(1$ st, 2nd and 3rd column respectively) with the virial velocity dispersion $\sigma_{\text {vir }}$. The first row is for the redshift bin $0.2<z<0.6$, and the second for $0.6<z<1.0$. In each plot, the upper panel shows isodensity contours in the plane $\sigma_{\text {meas }}$ versus $\sigma_{\text {vir }}$ for groups with at least 5 members. Blue triangles are the median (on $x$ axis) and mean (on $y$ axis) values of single points grouped in bins of $\sigma_{\text {vir }}$, with vertical error bars being the rms of mean values. The bins on the $x$ axis have the following limits: $0.5-150-250-350-450-550-1100 \mathrm{~km} \mathrm{~s}^{-1}$. Purple squares are the same as triangles but for groups with at least 8 members. The lower panel in each plot shows the percentage error (with its rms) when we compare $\sigma_{\text {meas }}$ and $\sigma_{\text {vir }}$. Triangles and squares are plotted only when the corresponding $\sigma_{\text {vir }}$ bin contains at least 15 objects. See text for further details.

where $\sigma_{\text {meas }}$ is $\sigma(100,0), \sigma(33,0)$, and $\sigma(33,275)$ in the three columns, respectively. Symbols have the same meaning as in the upper panel. follows:

Results graphically shown in Fig. 3 can be summarized as

1) Effects of the VVDS-02h flux limit. The plots in the first column show that even in the ideal case of purely flux-limited mock catalogues with $100 \%$ sampling rate and zero redshift measurement error, the measured velocity dispersion $\sigma(100,0)$ systematically underestimates $\sigma_{\text {vir }}$. This systematic offset, shown in the lower part of the plots, is always below $20 \%$, and its scatter decreases with increasing $\sigma_{\text {vir }}$ and is lower in the lower redshift bin. This offset can be easily understood by noting that in a flux-limited survey, even with a $100 \%$ sampling rate, higher redshift groups will progressively lose the fainter members that lie outside the selected flux range. As a consequence the measured velocity dispersion will underestimate the true virial velocity dispersion, because the detectable galaxies will be the brighter, that usually are found in group cores.

2) Effects of the lower sampling rate introduced by VVDS-02h strategy. The plots in the second column show that if we decrease the sampling rate from $100 \%$ to $33 \%$, our ability to recover $\sigma_{\text {vir }}$ decreases as well, as expected. The systematic offset is not significantly worse than in mocks with $100 \%$ sampling rate, but the scatter about the systematic offset is larger, especially for low $\sigma_{\text {vir }}$.

3) Effects of the VVDS redshift measurement error. Finally, the plots in the third column illustrate that when we add $275 \mathrm{~km} \mathrm{~s}^{-1}$ of a redshift error, low $\sigma_{\text {vir }}$ are very difficult to recover, while, for $\sigma_{\text {vir }}>350 \mathrm{~km} \mathrm{~s}^{-1}$, the systematic offset and its rms remain below $25 \%$ with a slightly higher scatter for the higher redshift bin.

Figure 3 convincingly demonstrates that the estimate of the velocity dispersion is robust only in groups with $\sigma_{\text {vir }}>350 \mathrm{~km} \mathrm{~s}^{-1}$. It also serves the following purpose: it forecasts the precision with which the measured velocity dispersion traces a specific $\sigma_{\text {vir }}$ of the matter particles in the halo once the VVDS sampling rate and spectroscopic uncertainties are taken into account. When we analyze the real VVDS-02h group catalogue, only the estimate $\sigma_{\text {meas }}$ are indeed available, and nothing is known about $\sigma_{\text {vir }}$. We should therefore ask also the converse question of how far a given value of $\sigma_{\text {vir }}$ is from the observed $\sigma_{\text {meas }}$. This means that we have to take $\sigma_{\text {meas }}$ as a reference when we compute the percentage error. We show the results of this analysis in Fig. 4 in which, for any given bin of $\sigma(33,275)$ we have plotted the mean 

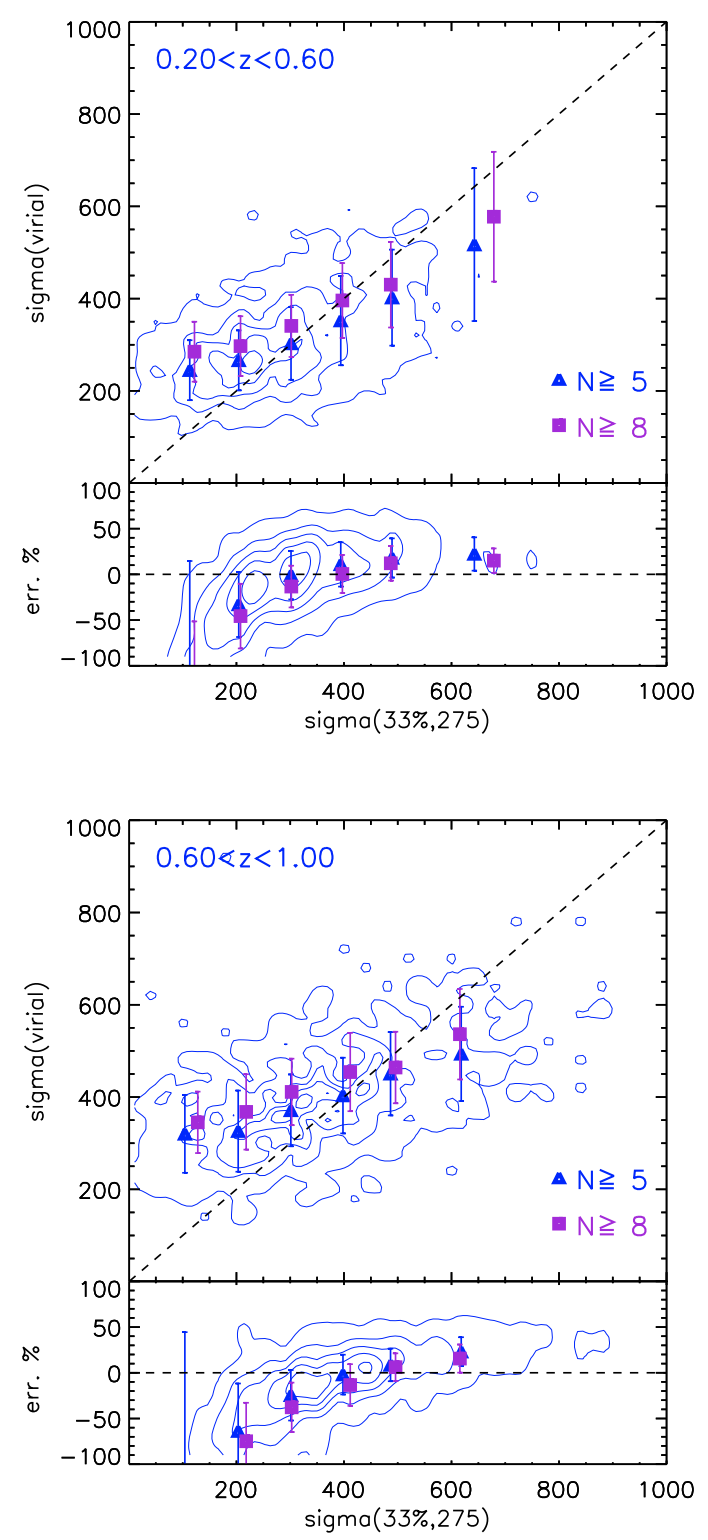

Fig. 4. As the plots in the last column of Fig. 3, but with exchanged axes. In this case, the binning is made according to $\sigma(33,275)$ and not $\sigma_{\text {vir. }}$.

systematic offset from the real underlying $\sigma_{\text {vir }}$, computed as a percentage error in $\sigma(33,275)$ as

err. $\%=\frac{\left[\sigma(33,275)-\sigma_{\text {vir }}\right]}{\sigma(33,275)} \times 100$.

When considering $\sigma(33,275)$ greater than $350 \mathrm{~km} \mathrm{~s}^{-1}$, in this case we are also able to recover $\sigma_{\text {vir }}$ with an error of $<30 \%$ and $<20 \%$ for groups with $N \geq 5$ and $N \geq 8$ respectively. Therefore, for velocity dispersions above $350 \mathrm{~km} \mathrm{~s}^{-1}$ and despite the relatively large error in redshift measurements causing a systematic increase in the estimated $\sigma(33,275)$, we can still determine a reliable value of $\sigma_{\text {vir }}$ in the VVDS-2h 4 pass region. We performed a similar check for groups in 1 pass and 2 pass regions, and verified that results are qualitatively similar to those obtained in the 4 pass area.

Our analysis globally suggests that we can use VVDS-02h data as a suitable sample for extracting high- $z$ groups.

\section{The group-finding algorithm}

Several geometrical algorithms have been proposed to identify groups and clusters from the 3-dimensional distribution of galaxies, that is by optically identifying them within spectroscopic redshift surveys (see Sect. 1).

In this work we identified groups using the VoronoiDelaunay method (VDM, Marinoni et al. 2002), which combines information about the three-dimensional Voronoi diagram and its dual, the Delaunay triangulation. The Voronoi diagram (Voronoi 1908) is a polyhedral partition of 3D space, each polyhedron surrounding a galaxy and defining the unique volume containing all the points that are closer to that galaxy than to any other galaxy in the sample. The Delaunay complex (Delaunay 1934) also contains proximity information. It is defined by the tetrahedra whose vertices are sets of four galaxies that have the property that the unique sphere that circumscribe them does not contain any other galaxy. The center of the sphere is a vertex of a Voronoi polyhedron, and each face of a Voronoi polyhedron is the bisector plane of one of the segments that link galaxies according to the Delaunay complex.

The basics of the VDM code are as follows. The denser the environment in which a galaxy lives, the smaller its associated Voronoi volume. Therefore the Voronoi partition performs a straightforward identification of the central regions of structures. In addition, the Delaunay triangulation assigns galaxy members to the identified core. We note that a crucial difference between the VDM and other methods is that, since it preliminarily identifies group centers, group membership reconstruction proceed radially outward, from the densest cores towards the outskirts of the structures.

An advantage offered by the Voronoi-Delaunay method is that it exploits the natural clustering of the galaxies in the sample. For example, the dimension of the volume assigned to each galaxy depends locally on the number density of the objects surrounding the galaxy itself. It is thus adaptively and unparametrically rescaled and not predefined on the basis of some fixed length parameter. Moreover, galaxies that are Delaunay connected to the central cores are processed with cylindrical windows whose dimensions are locally scaled on the basis of physical relations observed in simulated (and real) samples of groups and clusters. The specific set of VDM parameters is thus designed to provide the maximum amount of flexibility in selecting groups according to the a priori physical information we have about their structure. As a consequence, a fine-tuned VDM algorithm has been proven to be very efficient in reconstructing intrinsic characteristics of groups, such as for example the l.o.s. velocity dispersion of their members (Marinoni et al. 2002).

The Voronoi-Delaunay method was specifically designed to avoid some known drawbacks characterizing standard groupfinding algorithms such as for example the FOF and the hierarchical methods. These methods are based on user-specified parameters (the FOF linking length, the "affinity" threshold in the hierarchical method) that do not depend on the true distribution of galaxies. One of the negative consequences is that spatially closed but unrelated structures often merge to form a single system. Moreover, some dynamical properties of clusters are very sensitive to the adopted group-finding algorithm: for example, the velocity dispersion of groups identified by the FOF algorithm is found to be systematically higher (by nearly 30\%) than that of groups found by the hierarchical algorithm, even when both algorithms are optimized for the same galaxy sample (Giuricin et al. 2001). 
We briefly describe here the VDM, although detailed accounts can be found in Marinoni et al. (2002) and Gerke et al. (2005) (from which we adopted some technical improvements).

The algorithm first computes the Voronoi-Delaunay mesh following the prescriptions in Barber et al. (1996) and Mirtich (1996). It then searches for groups using a 3-step procedure. At each step, new group members are identified by means of a cylindrical window (of radius $R$ and half-length $L$ ), which is used to scan Delaunay-connected galaxies and decide whether or not they are cluster members. Phase I involves the 3-D identification of group seeds. In Phase II, the algorithm determines group central richness, and finally in Phase III an adaptive scaling based on the $\mathrm{N}-\sigma$ relation is used to rescale the cylinder dimensions depending on the group richness measured in Phase II. A detailed explanation of each of these three steps is given in Sects. 4.1, 4.2, and 4.3.

The radius and the half-length of the cylinders in both Phase I $\left(\mathcal{R}_{\mathrm{I}}\right.$ and $\mathcal{L}_{\mathrm{I}}$ ) and of Phase II $\left(\mathcal{R}_{\mathrm{II}}\right.$ and $\left.\mathcal{L}_{\mathrm{II}}\right)$, in addition to $r$ and $l$, the scaling factors used to determine respectively the radius and the half-length of the cylinder of Phase III $\left(\mathcal{R}_{\text {III }}\right.$ and $\left.\mathcal{L}_{\text {III }}\right)$, are free parameters of the algorithm. They need to be optimized using physical information about clusters.

The choice of a cylindrical shape for the search window is physically motivated by the gravitational field of galaxy overdensities inducing peculiar velocities whose effect is to make the galaxy distribution appear elongated in the redshift direction. The only way to take this into account is by using a search window with a radial extension much longer than the transversal dimension, in order not to miss group members. We note that we also use a cylindrical window during our Phase I, while in Marinoni et al. (2002) the Phase I search window had a spherical shape. The original choice of a spherical window during the first phase was physically motivated by the finding that galaxies residing in the highest density peaks, i.e. the central cores of groups and clusters, are expected to have smaller peculiar velocities. However, we verified that for less rich systems, i.e. loose groups such as those we expect to recover in the VVDS sample, the optimal choice is a cylindrical window. The survey's quite large redshift measurement error and the sparse sampling rate were particularly important in deciding our choice.

As we wish the length of search cylinders to correspond roughly to the peculiar velocity of the galaxies in the group, we have to consider that the mapping between redshift interval and peculiar velocity changes with redshift, and thus, following Gerke et al. (2005), our algorithm automatically rescales cylinder lengths $\mathcal{L}(z)$ as a function of $z$, using the equation

$\mathcal{L}(z)=\left[s(z) / s\left(z_{0}\right)\right] \mathcal{L}\left(z_{0}\right)$,

where $z_{0}$ is a reference redshift (see Sect. 4.2 for details) and

$s(z)=\frac{1+z}{\sqrt{\Omega_{M}(1+z)^{3}+\Omega_{\Lambda}}}$.

This scaling is applied as a function of redshift to all $\mathcal{L}_{\mathrm{I}}, \mathcal{L}_{\mathrm{II}}$, and $\mathcal{L}_{\text {III. }}$

\subsection{Phase I}

During Phase I, galaxies are ranked according to the increasing size of their Voronoi volume. A cylinder of radius $\mathcal{R}_{\mathrm{I}}$ and half length $\mathcal{L}_{\mathrm{I}}$ is then centered on the galaxy with the smallest Voronoi volume. All galaxies inside the cylinder and Delaunayconnected with the central galaxy are considered group members and called first-order Delaunay neighbours. The central galaxy and its first-order Delaunay neighbours are assumed to be a group seed. When no other galaxies are in the cylinder, the central galaxy is rejected as a potential seed. Thus, the choice of $\mathcal{R}_{\mathrm{I}}$ and $\mathcal{L}_{\mathrm{I}}$ determines the final number of identified groups. At the end of this Phase the barycenter of the seed is computed using the positions of the central galaxy and its first-order Delaunay neighbours.

The algorithm then processes the full sequence of Phases for the found seed. After Phases II and III are complete, the whole procedure is reiterated by selecting from the sorted list the first galaxy not yet assigned to a group.

\subsection{Phase II}

In the second phase a different cylindrical window with radius $\mathcal{R}_{\mathrm{II}}$ and half length $\mathcal{L}_{\mathrm{II}}$ is centered on the barycenter determined in Phase I, and used to determine the central richness of the group. All galaxies within the Phase II cylinder and connected to the first-order Delaunay neighbours are called secondorder Delaunay neighbours, and are considered to be additional group members. The total number of group members after this phase (the central galaxy plus first- and second-order neighbours) is assumed to be the central richness $N_{\text {II }}$ of the group.

A reliable estimate of $N_{\text {II }}$ is important because it controls the adaptive search window used during Phase III (see below). On the one hand, considering only Delaunay-connected galaxies minimizes the inclusion of interlopers in $N_{\text {II. }}$ On the other hand, in a flux-limited survey such as VVDS, the $N_{\text {II }}$ distribution varies as a function of redshift, because of the variation in the luminosity limit with redshift. To ensure a uniform group population, $N_{\mathrm{II}}$ must be corrected as a function of $z$ :

$N_{\mathrm{II}}^{\text {corr }}(z)=N_{\mathrm{II}} \frac{\left\langle v\left(z_{0}\right)\right\rangle}{\langle v(z)\rangle}$,

where $z_{0}$ is the redshift zero point considered as reference, and $\langle v(z)\rangle$ is the comoving number density, which we calculated by smoothing the redshift distribution of the galaxy sample, and then dividing it by the differential comoving volume element at the considered redshift. In Gerke et al. (2005), $z_{0}$ is the lower limit to the DEEP2 galaxy redshift distribution $n(z)$, i.e. $z_{0}=0.7$. For the VVDS-02h sample, the lower limit to $n(z)$ is $z=0.2$, but at this redshift the volume covered by the VVDS-02h is small. Because of this, $\langle v(z=0.2)\rangle$ can be poorly constrained. Moreover, $\langle v(z)\rangle$ decreases very rapidly from $z=0.2$ to $z=1.0$. Thus we chose $z_{0}=0.7$ as a compromise between high statistics (it is roughly the peak of our $n(z)$ distribution) and not yet so large survey volume.

At the end of Phase II, the barycenter position is readjusted using all $N_{\text {II }}$ members.

\subsection{Phase III}

During Phase III, the algorithm reconstructs the full set of group members, using a new search window centered on the group barycenter determined at the end of Phase II with dimensions determined according to the following basic scaling relations.

Assuming that groups are singular isothermal spheres, at any given distance $r$ from the center the mass density distribution is related to the velocity dispersion by the equation $\rho(r)=\sigma^{2} /\left(2 \pi G r^{2}\right)$ (Binney \& Tremaine 1988). Since $M(r)=$ $4 \pi r^{3} \rho(r) / 3$, and when defining $r_{\text {vir }}$ to be the radius of a spherical volume within which the mean density is $\Delta_{\mathrm{c}}$ times the critical density at the considered redshift, we find that $M_{\text {vir }} \propto \sigma^{3}$, where 
$M_{\mathrm{vir}}=M\left(r_{\mathrm{vir}}\right)$ is the virial mass. The virial theorem implies that $M_{\text {vir }} \propto \sigma^{3} \propto R^{3}$. By applying the correlation between velocity dispersion and central richness, confirmed for loose groups up to massive clusters (for example, see Bahcall 1981), we obtain the chain of relations $N_{\mathrm{II}}^{\text {corr }} \propto M_{\mathrm{vir}} \propto \sigma^{3} \propto R^{3}$.

Accordingly, we used the central richness $N_{\text {II }}^{\text {corr }}$ of each group to define both the radius and the length of the cylindrical search window:

$\mathcal{R}_{\mathrm{III}}=r\left(N_{\mathrm{II}}^{\mathrm{corr}}\right)^{1 / 3}$,

$\mathcal{L}_{\text {III }}=l\left(N_{\text {II }}^{\text {corr }}\right)^{1 / 3}$,

where, $r$ and $l$ are normalization parameters to be optimized using simulations. We note that the adaptive search window of Phase III differs from group to group and that all galaxies enclosed within the cylinder are assumed to be additional group members, irrespectively of the order of their Delaunay connections. From now on, we call richness $N$ the final number of members assigned to each group at the end of Phase III.

\section{Optimizing the group-finding algorithm}

\subsection{Success criteria}

In this section we describe the optimization strategy that we devised to reconstruct groups in the most reliable and unbiased way, using VVDS-like mock catalogues. We applied the VDM algorithm to these catalogues, and compared the groups found by the algorithm with the groups present in the mocks identified by the same FOF identification number (see Sect. 2.2). From now on, we refer to FOF groups in the mocks as "fiducial" groups, while groups reconstructed by our algorithm are called "reconstructed" groups, or simply "VDM" groups.

There are two levels of success we are interested in: 1) success in finding groups, i.e. to establish the level of contamination by interlopers and fake groups, the percentage of missed galaxies and missed groups, and other statistics of this kind; 2) success in reproducing group properties, i.e. accurately measuring group properties on a group-by-group basis, and reproducing their statistical distribution as accurately as possible.

To test the VDM algorithm success in finding the fiducial groups present in the VVDS-like mocks, we used the following quality estimators (see also Marinoni et al. 2002; and Gerke et al. 2005, for more details):

- galaxy success rate $S_{\text {gal }}$ : fraction of galaxies belonging to fiducial groups that are identified members of reconstructed groups;

- interlopers fraction $f_{\mathrm{I}}$ : fraction of galaxies identified by the algorithm as members of reconstructed groups that are, however, interlopers;

- completeness $C$ : fraction of fiducial groups that are "successfully" identified in the reconstructed catalogue;

- purity $P$ : fraction of reconstructed groups that "correspond" to fiducial groups.

Hence, we need to quantitatively determine whether a fiducial group is detected "successfully" and a reconstructed group "corresponds" to a fiducial one. We consider a detection to be successful when more than half of a fiducial group's members are detected in the same VDM group. In contrast, a VDM group corresponds to a fiducial one when more than half of its members belongs to that fiducial group. In general, these two conditions can be verified independently. These general cases are called one-way matches from one group catalogue to the other (from fiducial to VDM or in the opposite direction). But when these conditions are verified simultaneously involving the same fiducial and VDM group in both directions, we have a two-way match. We can therefore have a one-way completeness $\left(C_{1}\right)$ and a one-way purity $\left(P_{1}\right)$ when we consider only one-way matches in both the fiducial and the reconstructed group catalogue, respectively. A two-way completeness $\left(C_{2}\right)$ and a two-way purity $\left(P_{2}\right)$ can however also be defined when considering two-way matches.

On the one hand, knowing the absolute value of completeness and purity will help us to optimize the algorithm. On the other hand comparing, $C_{1}$ with $C_{2}$ and $P_{1}$ with $P_{2}$ we can establish the kind of errors in the reconstructed group catalogue. When $C_{1} \gg C_{2}$, it means that some fiducial groups are oneway successes but not two-way matches, and thus these fiducial groups contain a low fraction of the members of their reconstructed associated group. This is an indication that the VDM algorithm tends to overmerge separated groups to larger reconstructed groups, or to assign to reconstructed groups too many interlopers. On the other hand, when $P_{1} \gg P_{2}$ we know that VDM algorithm is affected by the opposite problem, i.e. the reconstructed groups are highly fragmented with respect to the fiducial ones.

We decided to use these indicators to search for the optimal parameter set for our algorithm following some guide lines. The basic idea is to obtain as high as possible values of $C_{1}$ and $C_{2}$, while keeping $P_{1}$ and $P_{2}$ at least above $50 \%$. We also attempted to produce neither a highly overmerged $\left(C_{1} \gg C_{2}\right)$ or a highly fragmented $\left(P_{1} \gg P_{2}\right)$ catalogue, and therefore we tried to ensure that $C_{1} \approx C_{2}$ and $P_{1} \approx P_{2}$.

\subsection{Algorithm optimization}

We applied the VDM algorithm to 20 VVDS-like mocks, obtaining group catalogues for the full redshift range $0.2 \leq z \leq 1.5$, but for the reasons discussed in Sect. 3.1 we implemented the optimization strategy only in the range $0.2 \leq z \leq 1.0$.

With a trial and error approach, we explored the flexibility of the 6 VDM parameters in recovering groups in a robust way. We allowed each parameter to vary across a comprehensive range. In particular, 1) we allowed $\mathcal{R}_{\mathrm{I}}$ and $\mathcal{R}_{\mathrm{II}}$ to increase to $1 h^{-1} \mathrm{Mpc}$, with no lower limit: this was because we defined the radii to span projected dimensions up to typical central radius of massive clusters (Bahcall 1981). 2) We allowed $r$ to span the range $0.4 \leq r \leq 1.5$, as we wished the radius of the last search cylinder to be equal or larger than small group typical size $\left(\sim 0.5 h^{-1} \mathrm{Mpc}\right.$, see Borgani et al. 1997 and references therein) and smaller than an Abell radius ( $\sim 1.5 h^{-1} \mathrm{Mpc}$, see Borgani et al. 1997). 3) We defined $\mathcal{L}_{\mathrm{I}}, \mathcal{L}_{\mathrm{II}}$, and $l$ to vary from 4 to $20 h^{-1} \mathrm{Mpc}$, to include clusters with velocity dispersions as high as $2000 \mathrm{~km} \mathrm{~s}^{-1}$ also at high redshift $(z \sim 1)$. In this case, the lower limit is determined mainly by our redshift measurement error, which has to be added to peculiar velocities. We imposed on $\mathcal{R}_{\text {III }}$ and $\mathcal{L}_{\text {III }}$ the same limits applied to $r$ and $l$. Nevertheless, we also checked the performances of the algorithm when no limits are applied to $\mathcal{R}_{\text {III }}$ and $\mathcal{L}_{\mathrm{III}}$, and we verified that, with the exception of very few cases, $\mathcal{R}_{\text {III }}$ and $\mathcal{L}_{\text {III }}$ "behave well", as we expected because the whole algorithm is based on physical scales and scaling laws.

Exploring the $6 \mathrm{D}$ parameter space, we found the parameter set that kept $C_{1}$ and $C_{2}$ as high as possible and $P_{1}$ and $P_{2}$ at least above $50 \%$, while we monitored the behavior of the group properties, both on a group-by-group basis and from a statistical point 

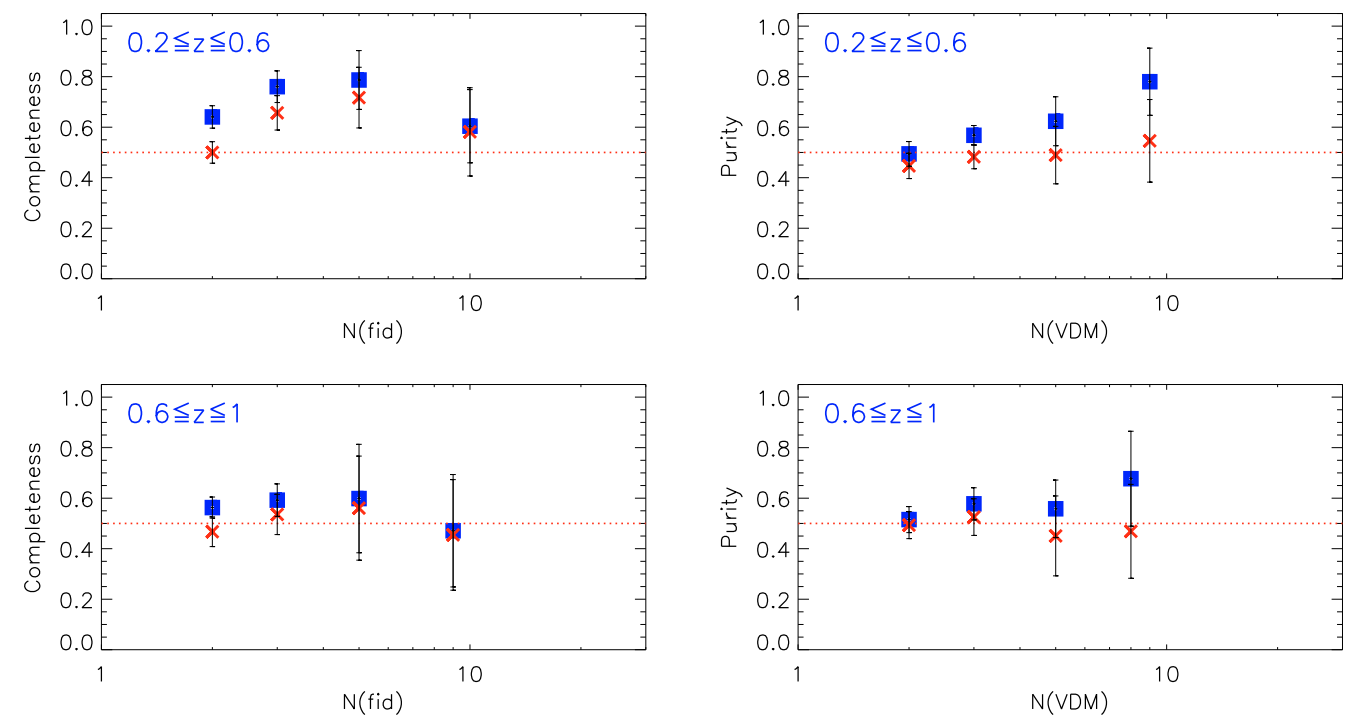

Fig. 5. $C_{1}$ and $C_{2}$ statistics as a function of "fiducial" group members (on the left) and $P_{1}$ and $P_{2}$ statistics as a function of "reconstructed" group members (on the right). One-way statistics are shown as blue squares, and two-way statistics as red crosses. $C$ and $P$ have been computed separately in each mock: in these plots, points are $C$ and $P$ values averaged over all mocks, while error bars are their rms. On the $x$ axis, we grouped the number of elements in the following way: $[N=2],[N=3,4],[N=5,6]$, and $[N \geq 7]$.

of view. We then moved slightly around these chosen values in smaller steps, to search for a possible finer tuning.

At the end of this finer search, we found the following parameter set, from now on called the best set of parameters

$-\mathcal{R}_{\mathrm{I}}=0.28 h^{-1} \mathrm{Mpc}$

$-\mathcal{L}_{\mathrm{I}}=7.0 h^{-1} \mathrm{Mpc}$

$-\mathcal{R}_{\mathrm{II}}=0.6 h^{-1} \mathrm{Mpc}$

$-\mathcal{L}_{\mathrm{II}}=5.0 h^{-1} \mathrm{Mpc}$

$-r=0.55 h^{-1} \mathrm{Mpc}$

$-l=14.0 h^{-1} \mathrm{Mpc}$.

We assigned to each group a redshift and a position in the $R A$ Dec plane, defined, respectively, as the median values of redshift, right ascension and declination of the group members.

Values of the quality parameters $C_{1}, C_{2}, P_{1}, P_{2}, S_{\text {gal }}$ and $f_{\mathrm{I}}$ can be found in Table 1 . We note that, to test the quality of the algorithm as a function of redshift, we separately considered two redshift bins $(0.2 \leq z \leq 0.6$ and $0.6 \leq z \leq 1.0)$.

We also analyzed completeness and purity as a function of group richness. Figure 5 shows $C_{1}$ and $C_{2}$ as a function of "fiducial" group members and $P_{1}$ and $P_{2}$ as a function of "reconstructed" group members. One-way statistics are shown as blue squares, and two-way statistics are shown as red crosses. The parameters $C$ and $P$ were computed separately in each mock. In Fig. 5, we plot $C$ and $P$ values averaged over all mocks, while error bars are their rms. The differences between $C_{1}$ and $C_{2}$ and between $P_{1}$ and $P_{2}$ indicate that our group catalogue is completely free from neither overmerging nor fragmentation. Figure 6 also indicates that, while the galaxy success rate $S_{\text {gal }}$ does not vary much as a function of $N$, the interloper fraction $f_{\mathrm{I}}$ decreases by a factor of $\sim 2$ from $N \geq 2$ to $N \geq 9$.

\subsection{Tests on recovered group properties}

As discussed above, the comparison between group properties in both the fiducial and the reconstructed catalogue is an important way of verifying that the VDM algorithm is not only
Table 1. Quality statistics $\left(C_{1}, C_{2}, P_{1}, P_{2}, S_{\text {gal }}\right.$, and $f_{\mathrm{I}}$, see text for details) of the reconstructed group catalogue, for two different redshift bins and the whole redshift range.

Quality statistics for $N \geq 2$

\begin{tabular}{cccc}
\hline \hline Quality parameter & $0.2 \leq z \leq 0.6$ & $0.6 \leq z \leq 1.0$ & $0.2 \leq z \leq 1.0$ \\
\hline$C_{1}$ & $0.68 \pm 0.03$ & $0.57 \pm 0.04$ & $0.63 \pm 0.03$ \\
$C_{2}$ & $0.56 \pm 0.04$ & $0.49 \pm 0.05$ & $0.53 \pm 0.03$ \\
$P_{1}$ & $0.56 \pm 0.02$ & $0.55 \pm 0.04$ & $0.56 \pm 0.02$ \\
$P_{2}$ & $0.48 \pm 0.04$ & $0.50 \pm 0.04$ & $0.49 \pm 0.03$ \\
$S_{\text {gal }}$ & $0.72 \pm 0.03$ & $0.59 \pm 0.03$ & $0.67 \pm 0.02$ \\
$f_{\mathrm{I}}$ & $0.38 \pm 0.02$ & $0.43 \pm 0.04$ & $0.40 \pm 0.02$ \\
\hline
\end{tabular}

Quality statistics for $N \geq 3$

\begin{tabular}{cccc}
\hline \hline Quality parameter & $0.2 \leq z \leq 0.6$ & $0.6 \leq z \leq 1.0$ & $0.2 \leq z \leq 1.0$ \\
\hline$C_{1}$ & $0.73 \pm 0.06$ & $0.57 \pm 0.05$ & $0.67 \pm 0.04$ \\
$C_{2}$ & $0.65 \pm 0.06$ & $0.52 \pm 0.06$ & $0.60 \pm 0.03$ \\
$P_{1}$ & $0.61 \pm 0.03$ & $0.58 \pm 0.05$ & $0.60 \pm 0.03$ \\
$P_{2}$ & $0.50 \pm 0.05$ & $0.51 \pm 0.07$ & $0.50 \pm 0.05$ \\
$S_{\text {gal }}$ & $0.75 \pm 0.04$ & $0.60 \pm 0.04$ & $0.70 \pm 0.03$ \\
$f_{\mathrm{I}}$ & $0.35 \pm 0.03$ & $0.40 \pm 0.05$ & $0.37 \pm 0.02$ \\
\hline
\end{tabular}

Notes. The first table shows $C_{1}, C_{2}, P_{1}, P_{2}, S_{\text {gal }}$, and $f_{\mathrm{I}}$ for all groups, while the second table shows these parameters for groups with at least 3 members. Each parameter is computed as the mean over the 20 VVDS-like mocks, and the associated error is its rms.

able to recover real groups, but also to maintain their characteristics. This means that when we compare the two catalogues on a group-by-group basis, the fractions of interlopers and missing galaxies modify group properties only below some tolerance level. The same has to hold also for the fiducial and reconstructed statistical distributions of these properties, when considering that the reconstructed catalogue contains fake groups and it fails to detect some groups. In this section, we show the properties of the redshift and velocity dispersion distributions for both the fiducial and reconstructed group catalogue. 


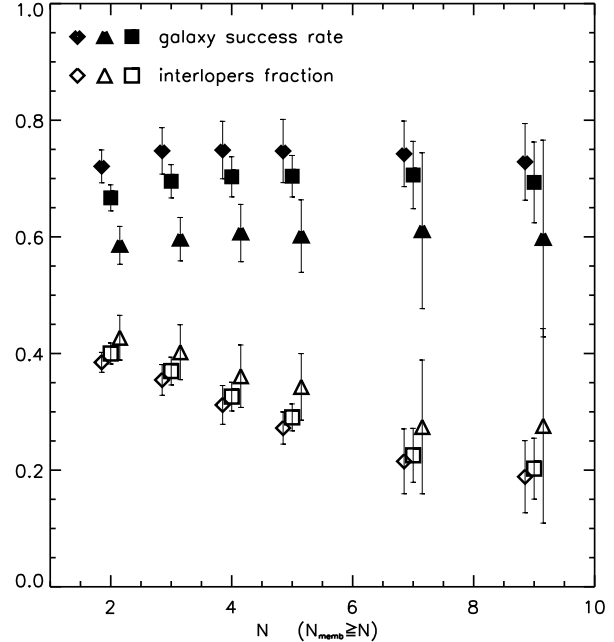

Fig. 6. Interlopers fraction $f_{\mathrm{I}}$ (empty symbols) and galaxy success rate $S_{\text {gal }}$ (full symbols) for different lower limits of group richness ( $x$ axis). Diamonds are for the redshift bin $0.2 \leq z \leq 0.6$, triangles for $0.6 \leq z \leq$ 1.0 , and squares for the entire range $0.2 \leq z \leq 1.0$. The parameters $S_{\text {gal }}$ and $f_{\mathrm{I}}$ have been computed separately in each mock. In this plot, points are $S_{\text {gal }}$ and $f_{\mathrm{I}}$ values averaged over all mocks, while error bars are their rms.

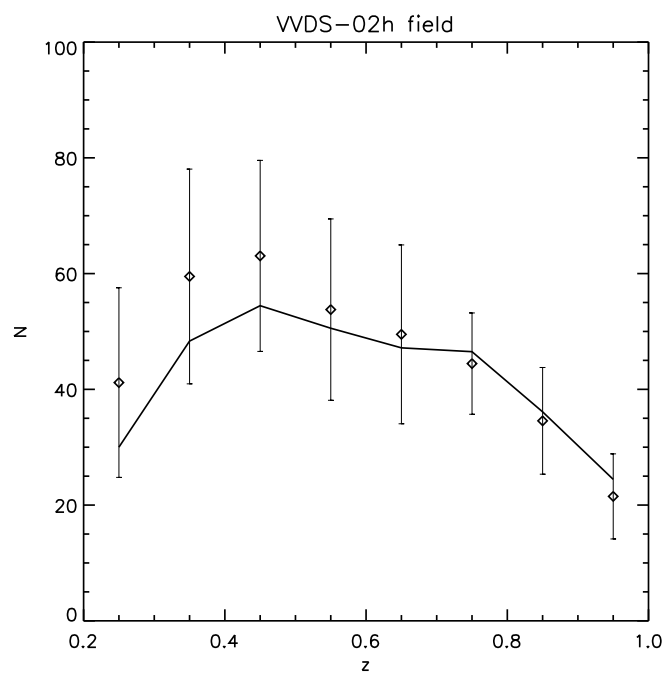

Fig. 7. Mean distribution of fiducial groups as a function of redshift (continuous line), computed as the average over 20 VVDS-like mocks. The mean distribution of VDM-reconstructed groups over the same 20 mocks is over plotted as black points.

\subsubsection{The $n(z)$ distribution}

We analyzed how well the "fiducial" groups redshift distribution $n_{\text {fid }}(z)$ is recovered by the distribution $n_{\mathrm{VDM}}(z)$ of the groups found by the algorithm. We averaged the $n_{\text {fid }}(z)$ distribution over 20 independent VVDS-like mocks to obtain its mean value, which is plotted as a continuous line in Fig. 7. In this figure, the mean $n_{\mathrm{VDM}}(z)$ for the same 20 independent mocks is shown as black points, the vertical bars being the rms for the 20 mocks. The plot shows that the difference between $n_{\mathrm{VDM}}(z)$ and $n_{\mathrm{fid}}(z)$, despite the presence of fake and/or missing groups in the VDM catalogue, is within the errors. A $\chi^{2}$ test between the two mean distributions infers that $\chi^{2}=1.4$. We therefore conclude that the two $n(z)$ distributions are statistically consistent with each other,

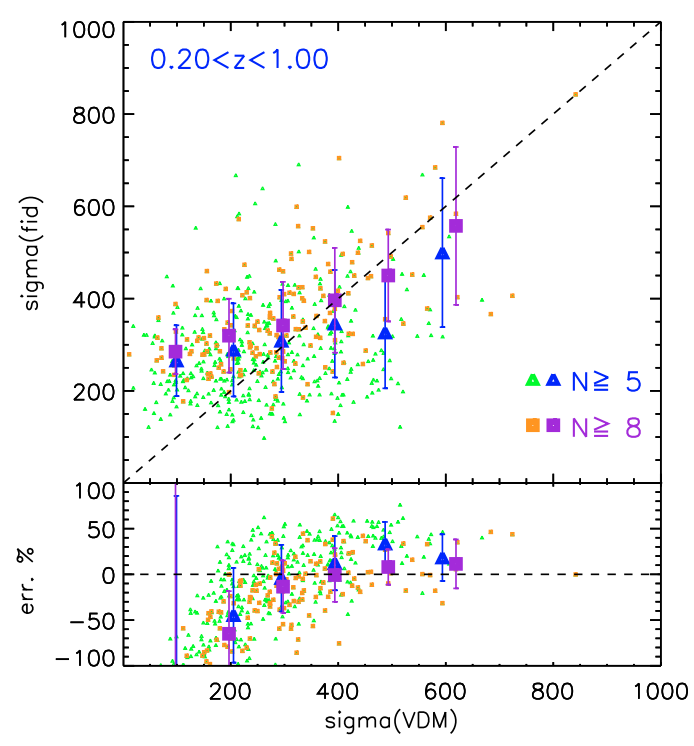

Fig. 8. Comparison between virial ( $y$ axis) and VDM reconstructed ( $x$ axis) group velocity dispersion. Only two-way matches are considered in this plot. The upper panel shows the scatter plot, the lower shows the percentage error. Green and blue triangles are groups with at least 5 members, orange and purple squares groups with at least 8 members; green and orange points are single groups, while blue and purple symbols are the median (on $x$ axis) and mean (on $y$ axis) values in bins of the property on the $x$ axis. Vertical error bars are rms of mean values.

even if there is a tendency for there to be more VDM reconstructed groups at low redshift. We repeated the same test using only groups with at least 5 members and with $\sigma \geq 350 \mathrm{~km} \mathrm{~s}^{-1}$, which are those groups for which we are sure we can compute a reliable velocity dispersion, and we also found in this case that $n_{\mathrm{VDM}}(z)$ and $n_{\mathrm{fid}}(z)$ are consistent with each other.

\subsubsection{Velocity dispersion}

For each VDM group, we measured the velocity dispersion $\sigma_{\text {VDM }}$ of its galaxies using Eq. (1), correcting it as indicated in Eq. (3). Figure 8 compares the velocity dispersion in the reconstructed groups $\left(\sigma_{\mathrm{VDM}}\right)$ with the virial velocity dispersion $\left(\sigma_{\mathrm{vir}}\right.$ quoted in the simulations) of the fiducial groups in VVDS-like mocks on a group-by-group basis. Only two-way matches are considered. The figure is divided into two panels as in Fig. 4: the upper part shows the scatter plot, the lower the percentage error, computed as in Fig. 4. Green and blue triangles are groups with at least 5 members, and both orange and purple squares represent groups with at least 8 members; green and orange points are single groups, while blue and purple symbols are the median (on $x$ axis) and mean (on $y$ axis) values in bins of the property on the $x$ axis. Vertical error bars are the rms of the mean values.

This scatter plot shows the following: on a group-by-group basis, for $\sigma_{\mathrm{VDM}} \geq 350 \mathrm{~km} \mathrm{~s}^{-1}$, close to the intrinsic limit set by the flux-limited nature of the VVDS catalogue, the correlation between $\sigma_{\mathrm{VDM}}$ and $\sigma_{\text {vir }}$ is such that $\sigma_{\mathrm{VDM}}$ overestimates $\sigma_{\text {vir }}$, but on average always by $\lesssim 30 \%$ for groups with at least 5 members, while this overestimate is on average $\lesssim 10 \%$ for groups with at least 8 members. As shown in Sect. 3.2, the velocity dispersion $\sigma_{\text {meas }}$ that one can measure in groups within a VVDS-like data sample is not a reliable estimator of $\sigma_{\text {vir }}$ for $\sigma_{\text {meas }} \leq 350 \mathrm{~km} \mathrm{~s}^{-1}$. 


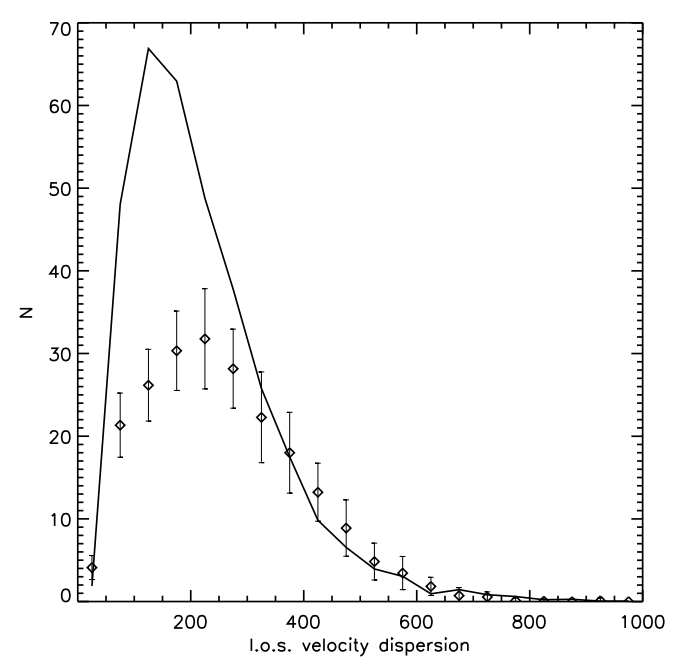

Fig. 9. Mean distribution of virial l.o.s. velocity dispersion (continuous line), computed as the average over 20 VVDS-like mocks. The mean distribution of $\sigma$ of groups reconstructed by the VDM, averaged over the same 20 mocks, is over plotted as black points, the vertical bars corresponding to its rms.

Besides the group-by-group comparison, it is also interesting to analyze the velocity dispersion distributions, thus including unrecovered and fake groups in the fiducial and reconstructed catalogues, respectively. Figure 9 compares the $n\left(\sigma_{\text {vir }}\right)$ and $n\left(\sigma_{\mathrm{VDM}}\right)$ distributions (the solid line and the black diamonds, respectively). The values on the $y$ axis are averaged over 20 VVDS-like mocks. The vertical bars associated with the $\sigma_{\text {VDM }}$ points represent their rms over the 20 mocks. We note that the areas below the two distributions differ. This is mainly because for the $\sigma_{\mathrm{VDM}}$ distribution we excluded groups for which we were unable to measure $\sigma$, i.e. groups for which we decided that $\sigma_{\mathrm{VDM}}=0$. This comparison indicates that the two distribution agree for $\sigma \geq 350 \mathrm{~km} \mathrm{~s}^{-1}$, as confirmed by a $\chi^{2}$ test between the two mean distributions for $\sigma \geq 350 \mathrm{~km} \mathrm{~s}^{-1}$.

As an additional test of the accuracy of the recovered $\sigma_{\mathrm{VDM}}$ distribution, we compared it with the $n\left(\sigma_{\text {vir }}\right)$ in mock catalogues with the same flux limits as the VVDS-02h sample but with $100 \%$ sampling rate (the $M(100,0)$ catalogues presented in Sect. 2.2), and the $n\left(\sigma_{\text {vir }}\right)$ of mock catalogues with no flux limits (the complete light cones from which the $M(100,0)$ catalogues were extracted). In Fig. 10, we show the normalized mean $n\left(\sigma_{\mathrm{VDM}}\right)$ (black diamonds) for $\sigma \geq 350 \mathrm{~km} \mathrm{~s}^{-1}$. It is the same distribution as in Fig. 9, but is normalized by the total numbers of groups with $\sigma \geq 350 \mathrm{~km} \mathrm{~s}^{-1}$. Overplotted green triangles represent the normalized mean $n\left(\sigma_{\text {vir }}\right)$ of fiducial groups in $M(100,0)$ mock catalogues, and the orange crosses are the normalized mean $n\left(\sigma_{\text {vir }}\right)$ distribution of fiducial groups in complete light cones of the Millennium Simulation. For each distribution, the redshift range considered is $0.2 \leq z \leq 1.0$. For these normalized distributions with $\sigma \geq 350 \mathrm{~km} \mathrm{~s}^{-1}$, a $\chi^{2}$ test between the $n\left(\sigma_{\mathrm{VDM}}\right)$ and the $n\left(\sigma_{\mathrm{vir}}\right)$ for $M(100,0)$ catalogues implies that the two distributions are statistically in agreement. We obtain the same result when we apply the same test to $n\left(\sigma_{\mathrm{VDM}}\right)$ and $n\left(\sigma_{\mathrm{vir}}\right)$ for the complete catalogues. This means that the $n\left(\sigma_{\mathrm{VDM}}\right)$ of the groups reconstructed by our algorithm is unbiased with respect to the $n\left(\sigma_{\text {vir }}\right)$ of groups in the complete light cones.

We repeated the tests shown in Figs. 9 and 10 also using only groups with at least 5 members, and we found similar results.

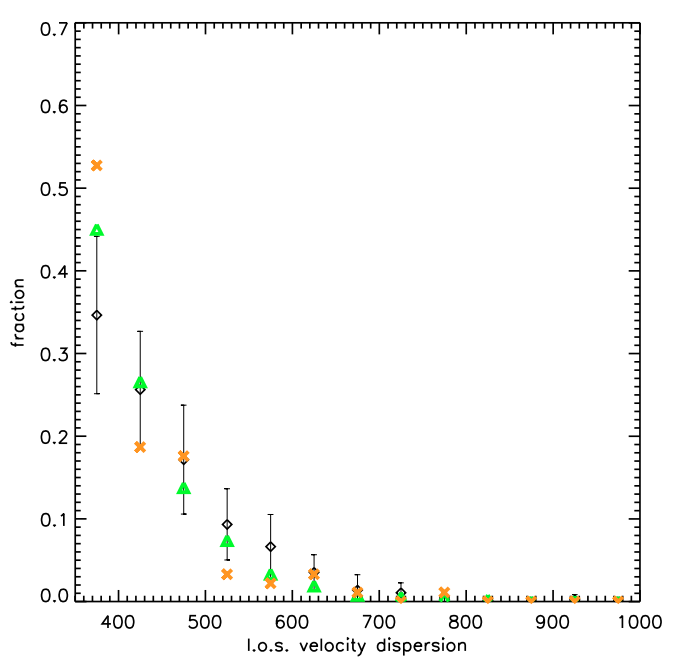

Fig. 10. Normalized mean distribution of $\sigma_{\mathrm{VDM}}$ (black diamonds) for $\sigma \geq 350 \mathrm{~km} \mathrm{~s}^{-1}$. It is the same distribution as in Fig. 9, but normalized by the total number of groups with $\sigma \geq 350 \mathrm{~km} \mathrm{~s}^{-1}$. Overplotted green triangles represent the normalized mean $\sigma_{\text {vir }}$ distribution of fiducial groups in Millennium mock catalogue with flux limits at $I_{\mathrm{AB}}=24$ and with $100 \%$ sampling rate $(M(100,0)$ mock catalogues, see Sect. 2.2$)$, and the orange crosses are the normalized mean $\sigma_{\text {vir }}$ distribution of fiducial groups in complete light cones of the Millennium Simulation, i.e. catalogues with no flux limits. For each distribution, the redshift range considered is $0.2 \leq z \leq 1.0$.

As discussed in Sect. 4, one of the primary goals of the VDM is to be able to recover the virial l.o.s. velocity dispersion of group galaxies, at least above some minimum threshold. This is not achieved, for example, by other commonly used group-finding algorithms, such as the FOF method (see Sect. 4). The comparisons between the $n(\sigma)$ distributions of reconstructed and fiducial groups presented in this section show that this aim has been successfully obtained in a deep redshift survey such as VVDS, at least up to $z=1$. Moreover, the VVDS redshift measurement error and sampling rate imposed an a priori lower limit to a reliable measurement of the l.o.s. velocity dispersion of group galaxies ( $\sigma \geq 350 \mathrm{~km} \mathrm{~s}^{-1}$, see Sect. 3.2). We demonstrate in Figs. 9 and 10 that the finding group algorithm that we used can not only recover a reliable $n(\sigma)$ distribution above some minimum $\sigma$, but also does not worsen the minimum $\sigma$ threshold imposed by the survey strategy itself. This result was achieved thanks to the flexibility of the 6 VDM parameters. Each of them has a specific role in determining the choice of the group members, by means of an intuitive localization of group barycenters (Phase I), a reliable estimate of the central richness (Phase II), and an appropriate use of group scaling laws (Phase III).

\subsection{Sampling rate}

As we applied the algorithm to the VVDS-like mocks, we optimized it for the whole observed area $\left(\sim 0.5 \mathrm{deg}^{2}\right.$ each), irrespective of the varying sampling rate across the field. Nevertheless, we also tested how both completeness and purity change if computed separately in areas with very different sampling rate, covered by 1,2 , or 4 passes of the spectrograph (hereafter called " $1 \mathrm{p}$ ", " $2 \mathrm{p}$ ", and " $4 \mathrm{p}$ " areas). For this test, we assigned each group to the $1 \mathrm{p}, 2 \mathrm{p}$, or $4 \mathrm{p}$ area according to its RA-Dec position (computed as the median value of RA and Dec of the member galaxies), even if it extends over an area with either a sudden decrease 
Table 2. Quality statistics $\left(C_{1}, C_{2}, P_{1}, P_{2}, S_{\text {gal }}\right.$, and $f_{\mathrm{I}}$, see text for details) of the group catalogue reconstructed by the algorithm with the high-purity parameter set, for two different redshift bins and the whole redshift range, considering groups with $N \geq 2$.

Quality statistics for $N \geq 2$

\begin{tabular}{cccc}
\hline \hline Quality parameter & $0.2 \leq z \leq 0.6$ & $0.6 \leq z \leq 1.0$ & $0.2 \leq z \leq 1.0$ \\
\hline$C_{1}$ & $0.29 \pm 0.03$ & $0.20 \pm 0.03$ & $0.24 \pm 0.02$ \\
$C_{2}$ & $0.24 \pm 0.03$ & $0.17 \pm 0.02$ & $0.20 \pm 0.02$ \\
$P_{1}$ & $0.75 \pm 0.04$ & $0.73 \pm 0.06$ & $0.74 \pm 0.04$ \\
$P_{2}$ & $0.66 \pm 0.07$ & $0.69 \pm 0.06$ & $0.67 \pm 0.05$ \\
$S_{\text {gal }}$ & $0.32 \pm 0.03$ & $0.21 \pm 0.04$ & $0.28 \pm 0.03$ \\
$f_{\mathrm{I}}$ & $0.24 \pm 0.04$ & $0.27 \pm 0.05$ & $0.25 \pm 0.03$ \\
\hline
\end{tabular}

Notes. Each parameter is computed as the mean over the 20 VVDSlike mocks, and the associated error is its rms. The same parameters for groups with $N \geq 3$ are consistent, within error bars, with those presented here.

or increase in the sampling rate. Considering the whole redshift range $0.2 \leq z \leq 1.0$, in the $4 \mathrm{p}$ area we find that $C_{1}=0.67 \pm 0.04$, $C_{2}=0.54 \pm 0.04, P_{1}=0.55 \pm 0.03$, and $P_{2}=0.48 \pm 0.08$, while in the $(1+2) \mathrm{p}$ areas $C_{1}=0.58 \pm 0.04, C_{2}=0.52 \pm 0.04$, $P_{1}=0.57 \pm 0.03$, and $P_{2}=0.49 \pm 0.04$.

While the changes in $C_{2}, P_{1}$, and $P_{2}$ are within the error bars, we measure a larger difference in $C_{1}$ when we decrease the number of spectrograph passes, i.e., the sampling rate. Analyzing the dependence of $C$ and $P$ on group richness, we can add that in the $4 \mathrm{p}$ area completeness is higher even for $N \geq 5$. We also note that in $4 \mathrm{p}$ area there is a higher overmerging, especially for $N \leq 4$, while in the $(1+2) p$ area fragmentation is increased for $N \geq 5$.

\subsection{High purity parameters}

With the best set of parameters, we can obtain from VVDS-02h data a group catalogue with high completeness, even though it has been shown that only $\sim 50 \%$ of groups is pure. This means that each group identified by the algorithm has, on average, only a $50 \%$ probability of being a real group. It may be useful to identify the subsample of groups that has an even higher probability of being real. Thus, we optimized the group-finding algorithm a second time, in this case maximizing purity (but paying attention not to reduce the new recovered group catalogue to a few "super-secure" groups). The so-called high-purity parameter set was found to be

$-\mathcal{R}_{\mathrm{I}}=0.10 h^{-1} \mathrm{Mpc}$

$-\mathcal{L}_{\mathrm{I}}=5.0 h^{-1} \mathrm{Mpc}$

$-\mathcal{R}_{\mathrm{II}}=0.6 h^{-1} \mathrm{Mpc}$

$-\mathcal{L}_{\mathrm{II}}=5.0 h^{-1} \mathrm{Mpc}$

$-r=0.55 h^{-1} \mathrm{Mpc}$

$-l=14.0 h^{-1} \mathrm{Mpc}$.

Table 2 shows $C$ and $P$ for the high-purity parameter set. Necessarily, $C$ is very low, but now each group identified by the algorithm has $\sim 70 \%$ of probability of being real, and the interlopers fraction $f_{I}$ decreases from $\sim 40 \%$ to $\sim 25 \%$ with respect to the one obtained with the best set of parameters (see Table 1).

\section{VVDS-02h field group catalogue}

We applied the group-finding algorithm to the VVDS-02h sample described in Sect. 2.1, using the best set of parameters. We defined the redshift and the position in the RA-Dec plane of each

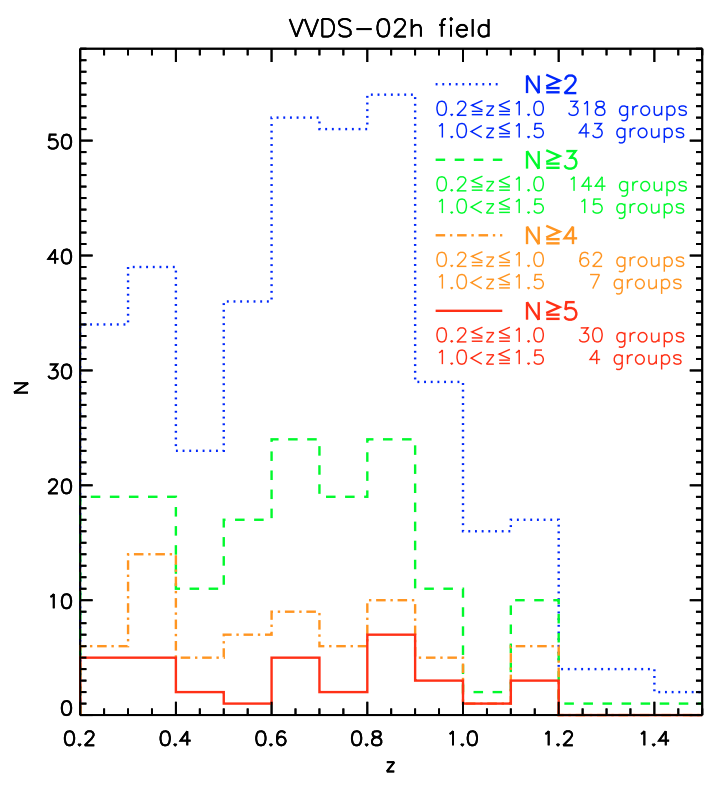

Fig. 11. The redshift distribution of groups in the VVDS-02h sample, found using the best set of parameters. Different line styles are for different cuts in group richness, as indicated. The total number of groups with the corresponding richness is quoted in the labels, for two different redshift ranges.

group as the median values of redshift, right ascension and declination of the group members. Figure 11 shows the redshift distribution of the identified groups, with different line styles for different cuts in group richness, as indicated in the figure. It is clear that beyond $z \sim 1$ there is a significant drop in the number of recovered groups, irrespective of their richness, as expected from Fig. 2. This drop in the redshift distribution may be partly related also to the choice of optimizing the algorithm only up to $z=1$ (see Sect. 5.2). We also applied the VDM to our galaxy sample using the high-purity set of parameters. With the best set of parameters, the algorithm identified 318 groups with 2 or more members in the redshift range $0.2 \leq z \leq 1.0$, one third of them having also been detected with the high-purity set. The identified groups comprise $\sim 19 \%$ of the galaxies in our sample. Comparing this percentage with the fraction of galaxies that reside in groups in VVDS-like mock catalogues, we found that it is consistent with both the fraction of galaxies residing in fiducial groups $(\sim 20 \%)$ and the percentage of galaxies residing in reconstructed groups $(\sim 22 \%)$.

For each group, we estimated the l.o.s. velocity dispersion $\sigma$. We used the gapper method, as described in Sect. 3.2, and we corrected it for the redshift measurement error by subtracting it in quadrature as in Eq. (3). We set $\sigma=0 \mathrm{~km} \mathrm{~s}^{-1}$ for those groups with a measured $\sigma_{\mathrm{G}}$ (from Eq. (1)) lower than the redshift error. $\sim 25 \%$ of groups with $\sigma \geq 350 \mathrm{~km} \mathrm{~s}^{-1}$ were detected by the algorithm also with the high-purity parameter set.

Given the small value of the parameter $r$, we note that, when driving the projected dimension of the search cylinder in Phase III (see Sect. 4.3), the typical projected radius within which the full set of group members is selected is always $<1 h^{-1} \mathrm{Mpc}$.

Detailed group catalogue statistics are shown in Table 3 . The number of groups found in VVDS-02h field is quoted. Different rows represent different values of velocity dispersion, different columns corresponding to different richnesses. The numbers in brackets indicate the number of groups that have been identified 
A\&A 520, A42 (2010)

Table 3. Number of VVDS-02h groups reconstructed by the algorithm using the best set of parameters in VVDS-02h field, for $0.2 \leq z \leq 1.0$.

\begin{tabular}{cccccccccc}
\hline \hline$\sigma\left(\mathrm{km} \mathrm{s}^{-1}\right)$ & \multicolumn{10}{c}{ Group members } \\
& 2 & 3 & 4 & 5 & 6 & 7 & 8 & 9 & ALL \\
\hline$\sigma=0^{a}$ & $89(23)$ & $24(10)$ & $8(2)$ & - & - & - & - & - & $121(35)$ \\
$0<\sigma<350$ & $61(25)$ & $39(18)$ & $18(6)$ & $8(5)$ & $3(2)$ & $3(3)$ & $2(1)$ & - & $134(60)$ \\
$\sigma \geq 350$ & $24(0)$ & $19(6)$ & $6(3)$ & $5(1)$ & $4(2)$ & $3(2)$ & $1(0)$ & $1(1)$ & $63(15)$ \\
\hline \multicolumn{1}{c}{} & \multicolumn{1}{c}{} & & & & Total: & $318(110)$ \\
\hline
\end{tabular}

Notes. Statistics are quoted as a function of the number of group members (columns) and measured l.o.s. velocity dispersion of group galaxies ( $\sigma$, in $\mathrm{km} \mathrm{s}^{-1}$, rows). Numbers in brackets indicate the number of groups also found by applying the algorithm with the high-purity set of parameters. ${ }^{(a)}$ We refer the reader to Sect. 6 for the meaning of $\sigma=0$.

Table 4. List of groups recovered in the VVDS-02h field in the range $0.2 \leq z \leq 1.0$. (This table is available in electronic form at the CDS).

\begin{tabular}{ccccccc}
\hline \hline grID & $\begin{array}{c}\text { RA } \\
\text { deg. }\end{array}$ & $\begin{array}{c}\text { Dec } \\
\text { deg. }\end{array}$ & $z$ & $N$ & $\begin{array}{c}\sigma \\
\mathrm{km} \mathrm{s}^{-1}\end{array}$ & Purity \\
\hline $124^{*}$ & 36.56310 & -4.31748 & 0.5850 & 3 & 351 & $\mathrm{H}$ \\
$144^{*}$ & 36.79910 & -4.59669 & 0.6135 & 5 & 428 & $\mathrm{H}$ \\
$224^{*}$ & 36.80323 & -4.67940 & 0.7898 & 4 & 392 & \\
\hline
\end{tabular}

Notes. Columns are the following: 1) group ID; 2) RA; 3 ) declination; 4) redshift; 5) number of detected members; 6) 1.o.s. velocity dispersion $\sigma ; 7)$ possible high purity. RA and declination are in degrees and $\sigma$ in $\mathrm{km} \mathrm{s}^{-1}$. RA, declination and redshift are the median values of all the galaxies in each group. The star near the group ID label those groups found by the algorithm also when using only galaxies with flag 3 and 4 (see text for further details). Column 7 labels with an " $H$ " those groups detected by the algorithm also with the high-purity set of parameters. See the electronic edition for the complete list of VVDS-02h groups.

by the algorithm also with the high-purity set of parameters (even if with fewer members).

We tested the reliability of the reconstructed catalogue by recomputing the groups excluding galaxies with flag $=2$ and 9 , i.e. using only galaxies whose redshift has a high likelihood $(>95 \%)$ of being correct. With respect to our original group catalogue, we verified that $80 \%(/ 77 \% / 75 \%)$ of the groups with at least 5 $(/ 4 / 3)$ members are still recovered. This implies that for these recovered groups the galaxies with flag $=2$ and 9 were not in the seed of the group, i.e. in the first set of galaxies recovered in Phase I of the algorithm (see Sect. 4.1).

Table 4 lists all the groups identified in the redshift window $0.2 \leq z \leq 1.0$. We note that the quoted number of members has to be assumed to be a lower limit to the true richness, as the sampling rate of our survey is not $100 \%$. The groups labeled with a star near their ID are those also recovered when using only galaxies with flag $=3$ and 4 . We apply this label only to groups with at least 3 members. The group members are presented in Table 5. We note that the galaxy ID is the same used to identify galaxies in the public VVDS release ${ }^{2}$.

In Fig. 12, we present the two-dimensional VVDS galaxy distribution, with galaxy positions projected in both RA and redshift. Each plot represents a different redshift bin, as indicated on the $y$ axis. Black dots are field galaxies, while coloured dots are group members (blue dots are pair members, green are triplet members, orange are quartet members and red dots are galaxies included in groups of 5 or more members).

\footnotetext{
${ }^{2}$ http://cencosw.oamp. fr
}
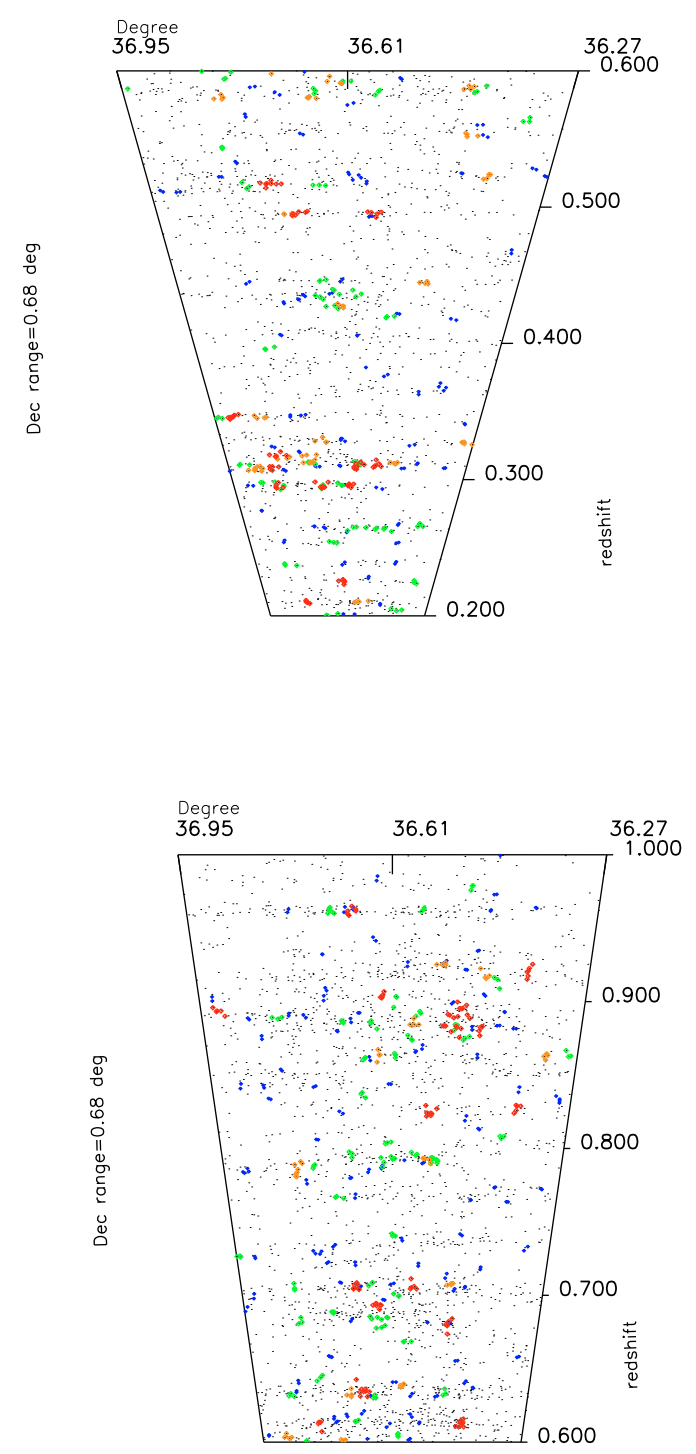

Fig. 12. Two-dimensional VVDS galaxy distribution as a function of Right Ascension and redshift (points are compressed on the Declination dimension). Each plot shows a different redshift bin $(0.2 \leq z \leq 0.6$ and $0.6 \leq z \leq 1.0$ in upper and lower panel respectively). Black dots are field galaxies, coloured dots are group members, according to the group catalogue obtained with the best set of parameters. The colour code is the following: blue dots are pair members, green are triplet members, orange are quartet members and red dots are members of groups with 5 or more members. 


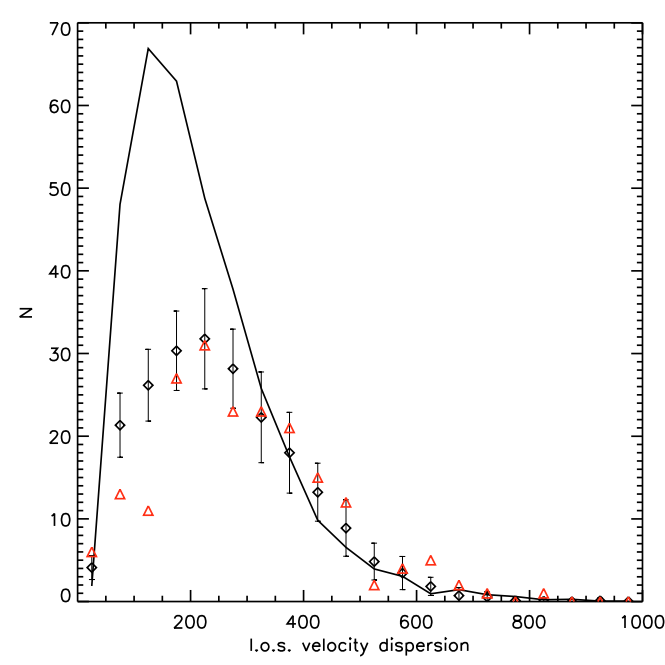

Fig. 13. As in Fig. 9, but in this case the velocity dispersion distribution of VVDS-02h field groups is also shown with red triangles.

Table 5. List of group galaxies belonging to the groups listed in Table 4. (This table is available in electronic form at the CDS).

\begin{tabular}{ccccccc}
\hline \hline galID $^{a}$ & $\begin{array}{c}\text { RA } \\
\text { deg. }\end{array}$ & $\begin{array}{c}\text { Dec } \\
\text { deg. }\end{array}$ & $z$ & $z$ flag & grID & $N$ \\
\hline 20309041 & 36.56095 & -4.31812 & 0.5859 & 4 & 124 & 3 \\
20309502 & 36.56310 & -4.31748 & 0.5850 & 4 & 124 & 3 \\
20310401 & 36.56771 & -4.31544 & 0.5824 & 2 & 124 & 3 \\
20176187 & 36.79656 & -4.61242 & 0.6072 & 4 & 144 & 5 \\
20183000 & 36.79910 & -4.59744 & 0.6135 & 4 & 144 & 5 \\
20183332 & 36.79801 & -4.59669 & 0.6136 & 4 & 144 & 5 \\
20184297 & 36.80199 & -4.59303 & 0.6137 & 2 & 144 & 5 \\
20184706 & 36.80420 & -4.59215 & 0.6126 & 3 & 144 & 5 \\
20146543 & 36.80323 & -4.68008 & 0.7857 & 3 & 224 & 4 \\
20146933 & 36.80903 & -4.67979 & 0.7890 & 3 & 224 & 4 \\
20147204 & 36.79638 & -4.67940 & 0.7911 & 4 & 224 & 4 \\
20151406 & 36.79351 & -4.66935 & 0.7898 & 3 & 224 & 4 \\
\hline
\end{tabular}

Notes. Columns are the following: 1) galaxy ID; 2) RA; 3) declination; 4) redshift; 5) redshift quality flag (see Sect. 2.1); 6) ID of the group to which the galaxy belongs 7) total number of group members. RA and Dec are in degrees. See the electronic edition of this article for the complete list of VVDS-02h group galaxies.

(a) The galaxy ID refers to the public VVDS release at

http://cencosw. oamp. fr

\subsection{L.o.s. velocity dispersion of group galaxies}

It is interesting to verify that the real universe is similar to the simulated one. Now we compare the VVDS catalogue with the Millennium-based mock catalogues.

We compared the $n(\sigma)$ distributions of real and simulated groups. Figure 13 shows the $n(\sigma)$ distribution for all VVDS$02 \mathrm{~h}$ groups in the redshift range $0.2 \leq z \leq 1.0$ (red triangles) and the $n(\sigma)$ distribution for VVDS-like mock catalogues. As in Fig. 9, the continuous line is the distribution of $\sigma_{\text {vir }}$ for fiducial groups, while black points represent the mean distribution for reconstructed groups, vertical bars being the rms of the 20 mock catalogues. In this plot, we consider the $\sigma$ measured with the gapper method (for both mocks and real data) and not the virial velocity dispersion. We exclude groups with measured $\sigma$ equal to 0 , because this value indicates that we have not been able to measure it due to the redshift measurement error (see Eq. (3)). This is the reason why the area under $n\left(\sigma_{\text {vir }}\right)$ in the plot is larger than the area under the other distributions. In this figure, we note the consistency between the $n(\sigma)$ distributions of true and mock group catalogues, at least for $\sigma>350 \mathrm{~km} \mathrm{~s}^{-1}$.

The relatively large number of groups for which the velocity dispersion estimated using Eq. (3) is formally negative is probably caused by our not taking into account possible dependences of the mean redshift error on the properties of the galaxies (i.e. magnitude, presence of emission lines etc.). It is most likely that for many of these groups the redshift error associated with their galaxy members is somewhat smaller than the adopted average value $\left(275 \mathrm{~km} \mathrm{~s}^{-1}\right)$. Nevertheless, we are reassured by none of the groups with $N \geq 5$ having $\sigma=0$.

\subsection{Comparison with other group catalogues in the same field}

Several group catalogues have already been compiled from different types of observations and with different methods in the sky area covered by the VVDS- $02 \mathrm{~h}$ field. For example, X-ray clusters have been identified from XMM-Newton images and then spectroscopically confirmed (Andreon et al. 2004b; Valtchanov et al. 2004; Andreon et al. 2005; Willis et al. 2005a,b; Bremer et al. 2006; Pierre et al. 2006). The matched-filter technique has also been used (Olsen et al. 2007) in addition to a weak lensing search (Gavazzi \& Soucail 2007) and structure identification using photometric redshifts (Mazure et al. 2007). All of these latter methods have been applied to photometric data from CFHTLS.

Among the X-ray clusters of the XMM-LSS, only 8 clusters fall in the VVDS-02h field area in the redshift bin $0.2 \leq$ $z \leq 1.0$ : XLSSC 005, XLSSC 013, and XLSSC 025 from the $\mathrm{C} 1$ catalogue, XLSSC 038 from the C2 catalogue and then the clusters $a, b, c$, and $d$ from the $\mathrm{C} 3$ catalogue (see Table 3 in Pierre et al. 2006). We find that both clusters $b$ and $c$ have a counterpart in our VDM catalogue (with 6 and 8 detected members, respectively) with an almost perfect match in their barycenters. Clusters XLSSC 013 and XLSSC 025 have possible counterparts at the same $z$ (with 4 and 3 members, respectively), but their barycenters in RA-Dec have a shift of $\sim 200 \mathrm{~h}^{-1} \mathrm{kpc}$. Inspecting these two groups in more detail, we find that the possible XLSSC 025 counterpart is dominated by a massive galaxy distant from the XLSSC 025 barycenter $\sim 100 h^{-1} \mathrm{kpc}$, which indicates that a closer match would have been obtained if we had computed a mass-weighted barycenter. In contrast, for a XLSSC 013 counterpart we do not identify any dominant galaxy. This shift of $\sim 200 h^{-1} \mathrm{kpc}$ could also be caused by the distribution the distances between the barycenters of VDM groups and their corresponding fiducial groups being a Gaussian centered at $\sim 0$ with a scatter of $\sim 200 h^{-1} \mathrm{kpc}$. Finally, we do not find counterparts for XLSSC 005, XLSSC 038, $a$, and $d$ in our catalogue. They fall inside our low sampling rate areas (i.e., those covered only by 1 or 2 passes of the spectrograph), and an additional inspection confirmed that the sampling rate in those regions does not allow our algorithm to find at least two galaxies inside the volume enclosed by the Phase I cylinder.

We concluded this comparison with XMM-LSS detections by inspecting the relation between optical and X-ray properties of the four groups for which there exists a (possible) XMM counterpart. In particular, we considered the relation between the X-ray luminosity $L_{X}$ presented in Table 5 of Pierre et al. (2006) and the velocity dispersions $\sigma$ that we measured. We verified that groups XLSSC 013, $b$ and $c$ have a $\sigma-L_{\mathrm{X}}$ relation in close agreement with the linear fit in the plane $\sigma$ - $L_{X}$ presented in Fig. 13 of Popesso et al. (2005). For group XLSSC 025, we measure a $\sigma$ that would be too low for its quoted $L_{\mathrm{X}}$, according 
to the indicated relation, but as its $\sigma$ is of the order of $200 \mathrm{~km} \mathrm{~s}^{-1}$ it does not reside in the $\sigma$ range that we consider to be reliably measured.

We note that our richest groups (10 groups with at least 7 members) do not correspond well to XMM-LSS clusters, except one that is the counterpart of the X-ray selected group $c$. There are at least three reasons why an optical group may not have been detected in X-rays: a) it may fall on the boundaries of a XMM-LSS pointing, thus in a region where the X-ray detector is affected by vignetting; $b$ ) it may have a redshift much higher than the mean $z$ reachable by the performed X-ray observations; c) it may have a low surface brightness, corresponding to a shallow potential well of the mass distribution, thus making $\mathrm{X}$-ray detection more difficult. We inspected our richest groups, and found that all of them fall in at least one of these three categories. In particular, we verified that the $N(z)$ distribution of all $\mathrm{X}$-ray clusters in the above-cited works is peaked at $z \sim 0.4$, while the $N(z)$ distribution of our richest groups is quite flat and reaches $z \sim 0.9$, there being 5 groups at $z \geq 0.7$. At least half of our richest groups also do not have a dominant member, that is a galaxy with luminosity and/or stellar mass much higher than the others. The VVDS-02h field sampling rate could be enough to explain this lack of dominant galaxies, but in principle we can not reject the hypothesis that a dominant galaxy in (some of) these groups may not exist, thus that these groups have a low $\mathrm{X}$-ray surface brightness.

We compared our group catalogue with those of Gavazzi \& Soucail (2007), Olsen et al. (2007), and Mazure et al. (2007).

Among the about 20 clusters in Olsen et al. (2007) inside the sky area and redshift range that we have explored, roughly half fall inside regions with too low sampling rate for our Phase I cylinder to be able to detect at least a pair; two of them (ID 30 and 42) fall very near in redshift to two wide structures at $z \sim$ 0.32 and $z \sim 0.45$, within which our algorithm detects (possibly fragmenting them) a few groups. Finally, considering the depth of the redshift bins in which Olsen's groups can reside $(\Delta z \sim 0.1)$ due to the use of photometric redshifts, we find that for 5 groups in Olsen's catalogue there exists a counterpart in our catalogue.

Among the about 30 structures detected by Mazure et al. (2007) in the redshift range $0.2<z<1.0$, we find that about 20 fall inside regions with too low a sampling rate for our finding group algorithm (13 of which are in the 1 pass area); a few of them reside in redshift slices $(z \sim 0.3, z \sim 0.7$, and $z \sim 0.9$ ) where a wide (in RA-Dec) structure is also present, which has possibly been fragmented by our algorithm, producing in our catalogue more than one counterpart. Finally, three of the structures detected by Mazure et al. (2007) have a possible direct counterpart in our catalogue (general ID 5, 19 and 21, see Table 3 in Mazure et al. 2007).

Finally, the 3 structures detected by Gavazzi \& Soucail (2007) that fall inside the VVDS-02h field are in very low sampling rate areas, thus in regions where our algorithm did not detect any group.

In this comparison, we also took into account that in the CFHTLS data used in the three above-mentioned works there are masked sky regions that have not been used for group finding, as shown for example in Fig. 1 in Mazure et al. (2007) and Fig. 9 in Olsen et al. (2007). We find that $\sim 5 \%$ of our groups in the range $0.2 \leq z \leq 1.0$ fall in those regions. Moreover, we observe that roughly half of this masked area falls inside the region that in the VVDS-02h field has the highest sampling rate (the central area highlighted in Fig. 1), and that this higher-sampling region covers only $\sim 25 \%$ of the VVDS- $02 \mathrm{~h}$ field. Thus, the percentage of our groups falling in the masked areas increases to $\sim 8 \%$ for groups with at least 3 members and to $20 \%$ for our 10 richest groups (those with at least 7 members).

\section{The $\boldsymbol{U}-\boldsymbol{B}$ colour of group galaxies}

After producing a catalogue of groups at high $z$, we wish to apply it to study the dependence of galaxy properties on environment and its evolution with cosmic time. More specifically, we investigate whether the physical properties of group galaxies differ from those of all galaxies, up to $z \sim 1$. Are the relations that we see in groups at low redshift already present at $z \sim 1$ ? Is there any unambiguous signature of time evolution in known scaling relations characterizing galaxies in cluster environments? In this paper, we do not carry on an exhaustive analysis of this topic, which is possibly the goal of future work. The main aim of this paper is to present the VVDS-02h field group catalogue and discuss its reliability. In this section, we show how our group catalogue can be used for studies related to environmental effects on galaxy properties on group scales.

As we wish to investigate the redshift evolution of the properties of group galaxies, we study a group sample that is homogeneous at all $z$. We thus require that the groups we use for this analysis have at least two members brighter than a luminosity limit that allows us to be complete up to $z=1$. This luminosity limit evolves with redshift. Following roughly the evolution of $M^{*}$, the characteristic magnitude of the luminosity function (Ilbert et al. 2005), we set this limit to be $M_{B} \leq-18.9-1.1 z$. Our "group galaxy" sample consists of galaxies brighter than this limit, in groups with at least two members brighter than this limit itself. Moreover, we define a "total" galaxy sample considering all galaxies brighter than this limit (including those also in groups).

After defining the sample, as a first step we studied the fraction of "blue" galaxies ( $f_{\mathrm{b}}$ from now on) in both the group and total samples, in the range $0.2 \leq z \leq 1.0$. The general blueing of cluster galaxy population for increasing redshift, first shown by Butcher \& Oemler (1978) and Butcher \& Oemler (1984) and known as the Butcher-Oemler effect, has been widely confirmed by subsequent studies (see for example Margoniner et al. 2001; De Propris et al. 2003; Gerke et al. 2007). Nevertheless, nowadays there is no full agreement about the origin of this blueing. It may be related to environmental effects (e.g. Dressler et al. 1997), but has also been proposed to be consistent with the overall ageing of all galaxies, irrespective of their environment (Andreon et al. 2004a, 2006).

According to our criteria, a galaxy is "blue" if it has a colour $U-B \leq 1$. This threshold has been chosen because it roughly corresponds to the minimum (i.e. the green valley) in the bimodal $U-B$ colour distribution. This colour cut was kept constant at all redshifts because we found that the green valley colour does not evolve much in the $z$ range considered. For the computation of the $U$ - and $B$-band absolute magnitudes, we refer the reader to Ilbert et al. (2005).

Since our goal is to study $f_{\mathrm{b}}$ as a function of redshift, we first verified that the failure rate in redshift measurement does not depend on redshift for specific galaxy colours. We assigned a "photometric type" to each galaxy according to the scheme proposed by Zucca et al. (2006). The classification is carried out by fitting the spectral energy distribution (SED) of galaxies to six templates (four observed spectra, Coleman et al. 1980, and two starburts SEDs, Bruzual \& Charlot 1993). We then proceeded as in Franzetti et al. (2007), by performing a bimodal classification. We considered E/S0 and early spirals as "early type", and late-type spirals, irregular and starburst types as "late-type". The 


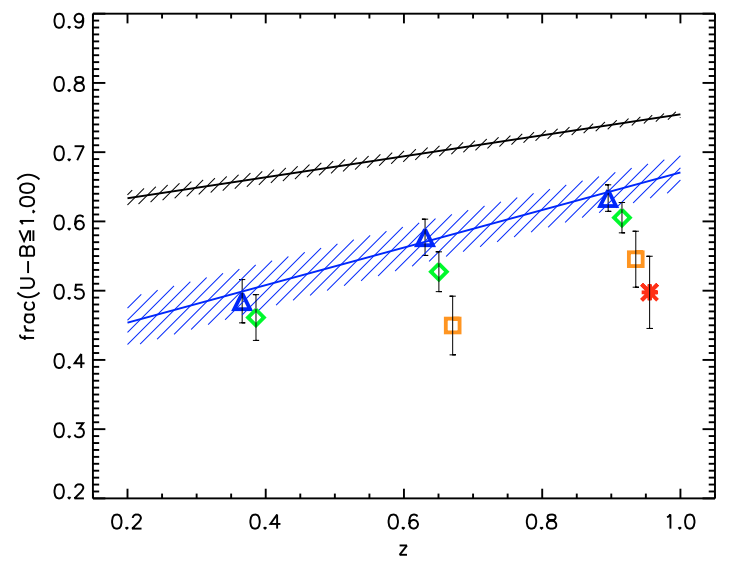

Fig. 14. Fraction of blue galaxies $(U-B \leq 1)$ for group galaxies (blue empty triangles) in three different redshift bins: $0.2 \leq z \leq 0.5,0.5 \leq z \leq$ 0.7 , and $0.7 \leq z \leq 1.0$. The linear fit to these three points is overplotted as a blue line, while the upper black line is the linear fit for $f_{\mathrm{b}}$ computed within the "total" sample. The dashed areas along the two linear fits show the locus where the linear fits could lie considering their 1- $\sigma$ error on both intercept and slope. See text for more details. Other symbols are for group galaxies in groups with increasing corrected richness: $N_{\text {corr }} \geq$ $7,14,20$ for green diamonds, orange squares, and red stars, respectively.

relation between this classification scheme and the colour $U-B$ that we used to compute $f_{\mathrm{b}}$ is monotonic, bluer colours being associated with "late type" templates. In particular, our "early type" population constitutes $>90 \%$ of the galaxies with $U-B>1$. We computed the "late type" galaxy fraction in both our spectroscopic sample and in the photometric parent catalogue, in three redshift bins in the range $0.1 \leq z \leq 1.0$ (using photometric redshifts for the parent catalogue, see Sect. 2.1 for their determination). As already found by Franzetti et al. (2007), who carried out a similar analysis on wider redshift intervals up to $z \sim 2$, the "late type" fraction is $3 \%$ higher in the spectroscopic sample and this increment does not depend on redshift. This result implies that any trend of $f_{\mathrm{b}}$ with redshift is not caused by a measurement bias in our sample.

Figure 14 shows $f_{\mathrm{b}}$ for the group galaxies (blue triangles) in three different redshift bins: $0.2 \leq z \leq 0.5,0.5 \leq z \leq 0.7$, and $0.7 \leq z \leq 1.0$. The vertical error bars are the $1 \sigma$ confidence levels associated with $f_{\mathrm{b}}$, computed with the usual approximation of the formula for binomial statistics given in Gehrels (1986) of $\sigma^{2}=f_{\mathrm{b}} f_{r} / n$, where $f_{r}=1-f_{\mathrm{b}}$ and $n$ is the total number of galaxies in the redshift bin.

As reference, we plot the linear fit of the three points as a blue line, while the upper black line is the linear fit for $f_{\mathrm{b}}$ computed within the "total" sample. The fraction $f_{\mathrm{b}}$ is clearly lower in groups than in the total sample. The slopes of the two fits and their $1 \sigma$ confidence levels are $0.27 \pm 0.07$ and $0.15 \pm 0.02$ for the group and total sample, respectively. Although they both differ significantly from zero, and the group slope is steeper, they are compatible with each other, the group sample slope being steeper only at a $1.6 \sigma$ significance level. At this stage of the investigation, we are only able to confirm the different overall value of $f_{\mathrm{b}}$ between group galaxies and the total sample, but not their possible different evolution. We verified that these results are insensitive to any variation in the $U-B$ threshold adopted to define blue galaxies (by $\pm 0.05 \mathrm{mag}$ ). We also found that they did not change significantly when we implemented a colour cut that depends on luminosity, following the mild dependence on magnitudes of the green valley locus. Although we did not detect any redshift dependence of the green valley locus up to $z=1$, we also allowed the colour cut to vary by 0.1 mag redward for any redshift decrease of $\mathrm{d} z=1.0$ (as suggested by Blanton 2006 and adopted by Gerke et al. 2007). Even in this case, the two slopes differ appreciably from zero, and the difference between them is significant at the $\sim 1.5 \sigma$ level.

We compared our results with those presented by Gerke et al. (2007), who studied the fraction of blue galaxies in both groups and the field within different subsamples extracted from the DEEP2 data set. In their sample I, that with a selection most similar to ours, they found that $f_{\mathrm{b}}$ is lower in groups than in the field, but they detected significant evolution in $f_{\mathrm{b}}$ with $z$ in neither groups nor the field. In all cases, evolutionary effects are much more difficult to quantify in that sample since the redshift range as well as the luminosity range covered is narrower with respect to that explored in this study.

Our results agree with those presented by Iovino et al. (2010), who studied the evolution of $f_{\mathrm{b}}$ in groups and the field within the zCOSMOS-10k sample (see also, for completeness, the analysis of Kovac et al. 2010, concerning the fraction of early-type galaxies in groups). This agreement is based on the comparison with their Sample II, that with a luminosity cut most similar to ours, and it holds for both the group and the total sample. Interestingly, Iovino et al. (2010) find that in their luminosity limited sample galaxy colour still depends on environment at $z \sim 1$ (with a trend similar to what we find in this work), but at the same redshift they do not converge to the same conclusion when the blue fraction is recovered from a (stellar) mass limited sample $\left(\log \left(M / M_{\odot}\right) \geq 10.8\right)$. They explain this result by suggesting that red galaxies of these stellar masses, already in place at $z \sim 1$, may be produced by internal mechanisms of evolution, on which environment has no influence. We refer the reader to Iovino et al. (2010) for more details.

Cucciati et al. (2006) carried on a similar analysis using the same VVDS-02h data set that we use in this work. They studied the colour-density relation up to $z=1.5$, with the local density computed within Gaussian filters with $\sigma=5 h^{-1} \mathrm{Mpc}$. They found that the colour-density relation becomes weaker for increasing redshift (the evolution of $f_{\mathrm{b}}$ being faster in high densities), and that at $z \sim 1$ no significant colour-density relation is detected, for galaxies with $M_{B} \leq-20$ (that is equivalent to the threshold we use in this work). Taken at face value, our results are not compatible with these previous findings, as we find that at $z \sim 1, f_{\mathrm{b}}$ still differs between groups and the total sample. This difference can be explained by our exploring higher densities/smaller scales $\left(<1 h^{-1} \mathrm{Mpc}\right.$, see Sect. 6). For example, several studies in the literature propose that environmental effects on large scales are only a weaker residual of those acting on smaller scales (e.g. Kauffmann et al. 2004; Blanton et al. 2006). The same hypothesis is suggested by Cooper et al. (2007), when comparing the colour-density relation found in the DEEP2 data set with the one presented in Cucciati et al. (2006). They still find a colour-density relation at $z \sim 1$, but on smaller scales than those investigated by Cucciati et al. (2006).

A direct comparison of results obtained on the basis of heterogeneous definitions of the local environment (as for example density field maxima as opposed to groups) is not straightforward. Cooper et al. (2007) showed, for example, that the evolution of the colour-density relation is continuous in the range $0.4<z<1.3$, while Gerke et al. (2007), who used the same DEEP2 data set but a different definition of environment based on groups, found that the evolution of $f_{\mathrm{b}}$ in groups is flat in the range $0.7<z<1.0$, and steepens for $1.0<z<1.3$. 
Nevertheless, the two works agree that at $z \sim 1.3$ the colourdensity relation seems to disappear. No need to emphasize that the physics associated with different environments has still to be fully understood.

A direct comparison of our results with those presented in other works up to $z \sim 1$ is not trivial. Interpretation is hampered by the the non-homogeneity of group catalogues selected according to different selection criteria. As a consequence, the picture emerging from these studies is complex and sometimes even contradictory. As we intend only to provide a general idea of the kind of studies that can be potentially carried out with our group sample, we do not enter into detail. We rather refer the reader to Poggianti et al. (2006) and Andreon et al. (2006) for a more in-depth discussion about the status of the art and the problems related to uncontrolled selection effects.

As a second step, we examined the behavior of $f_{\mathrm{b}}$ in groups characterized by different degrees of richness. There is still no agreement in the literature about the dependence of $f_{\mathrm{b}}$ on cluster properties. For example, $f_{\mathrm{b}}$ is found to both depend on cluster richness (Margoniner et al. 2001; Goto et al. 2003), or be independent of it, as well as of 1.o.s. velocity dispersion and mass (De Propris et al. 2004; Goto 2005; Popesso et al. 2007b). To address this issue, we associated each group with the number of members found by the algorithm. Nevertheless, due to the survey characteristics (sampling rate, spectra signal to noise ratio, etc.), the observed number has to be corrected to recover the real number of members within the flux limit of the survey $\left(I_{\mathrm{AB}} \leq 24\right)$. We did this by weighting each galaxies with the "target sampling rate" and the "spectroscopic success rate" of the survey (see Ilbert et al. 2005). We then modulated this mean weight with a finer correction taking into account that the sampling rate is not uniform in the field. This was done by using the $\Psi(\alpha, \delta)$ selection function described in Cucciati et al. (2006). For each group, we computed a corrected richness $\left(N_{\mathrm{c}}\right)$, which is the sum of the weights of those galaxies brighter than the evolving luminosity limit described above. In Fig. 14, green diamonds, orange squares, and red stars show $f_{\mathrm{b}}$ within groups with $N_{\mathrm{c}} \geq 7,14$, and 20 . In the plot, we can see a general trend of decreasing $f_{\mathrm{b}}$ for increasing $N_{\mathrm{c}}$, at any redshift explored. Given the error bars, this decrement is not significant when considering single steps in $N_{\mathrm{c}}$, but the overall tendency is clear. Nevertheless, the $f_{\mathrm{b}}$ redshift evolution does not appear to differ for different values of $N_{\mathrm{c}}$. These results are in agreement with those found by Iovino et al. (2010) in their analysis of the zCOSMOS-10k group sample, but we have extended them to fainter magnitudes. It would be indeed interesting to study the dependence on $N_{\mathrm{c}}$ of other galaxy properties, as done for example for star formation rate (SFR) and specific SFR (see for example Popesso et al. 2007b). This could provide insight into how different galaxies properties are affected by different environments. We defer this study to a future work.

\section{Summary and conclusions}

We have compiled a homogeneous catalogue of optical groups identified in the VVDS-02 field by means of the VDM algorithm, in the range $0.2 \leq z \leq 1.0$.

We used mock catalogues simulating the VVDS survey to optimize the performances of the group-finding algorithm (maximizing the completeness and the purity of the resulting group catalogue) as well as to minimize possible selection effects. Our main results are:

- Using the mock catalogues, we verified that the VVDS-02h survey sampling rate allows us to recover at least $50 \%$ of the groups (with a virial l.o.s. velocity dispersion $\sigma_{\mathrm{vir}} \geq$ $350 \mathrm{~km} \mathrm{~s}^{-1}$ ) that are potentially present in the parent photometric catalogue up to $z=1$.

- We tested how well $\sigma_{\text {vir }}$ of the halo mass particles can be estimated using sparsely sampled galaxy velocities. We verified that with this method, given the characteristics of our survey (flux limit, sampling rate, redshift measurement error) we are able to recover a sensible value of $\sigma_{\text {vir }}$ for $\sigma_{\text {vir }} \geq 350 \mathrm{~km} \mathrm{~s}^{-1}$.

- Applying the optimized algorithm to the VVDS real data set, we obtained a catalogue of 318 groups of galaxies (with at least two members) in the range $0.2 \leq z \leq 1.0$. Among these groups, 63 have a measured l.o.s. velocity dispersion greater than $350 \mathrm{~km} \mathrm{~s}^{-1}$. The group catalogue is characterized by an overall completeness of $\sim 60 \%$ and a purity of $\sim 50 \%$. Nearly $19 \%$ of the total population of galaxies live in these systems.

- The number density distribution as a function of both redshift $(n(z))$ and velocity dispersion $(n(\sigma))$ of the VVDS groups with $\sigma>350 \mathrm{~km} \mathrm{~s}^{-1}$ scales in qualitative agreement with the analogous statistics recovered from the mock catalogues.

- We studied the fraction $f_{\mathrm{b}}$ of blue galaxies $(U-B \leq 1)$ in the range $0.2 \leq z \leq 1$. We used a luminosity-limited subsample of galaxies extracted from our data $\left(M_{B} \leq-18.9-1.1 z\right)$, complete up to $z=1$. We found that $f_{\mathrm{b}}$ is significantly lower in groups than in the global galaxy population. Moreover, $f_{\mathrm{b}}$ increases as a function of redshift irrespective of the environment, with marginal evidence of a higher growth rate in groups. We also analyzed how $f_{\mathrm{b}}$ varies as a function of group richness, finding that, at any redshift explored, $f_{\mathrm{b}}$ decreases in systems with increasing richness.

Further explorations of the properties of VVDS groups is left to future work. We only anticipate that the high degree of completeness of the catalogue can be potentially exploited for extracting cosmological information via, for example, cluster count techniques. The high level of purity also makes the VVDS group sample ideal for astrophysical analyses studying various physical properties of galaxies as a function of local density and environment. We also note that the cross-correlation studies of our optically-selected catalogue with samples inferred in the same field with independent techniques will help us to gain insights not only into cluster selection biases but also the physics at work within these extreme environments.

Acknowledgements. O.C. thanks Stefano Andreon for stimulating discussions. We thank the referee for helpful comments. This research has been developed within the framework of the VVDS consortium and it has been partially supported by the CNRS-INSU and its Programme National de Cosmologie (France), by the Italian Ministry (MIUR) grants COFIN2000 (MM02037133) and COFIN2003 (num.2003020150) and by PRIN-INAF 2005 (CRA 1.06.08.10). The VLT-VIMOS observations have been carried out on guaranteed time (GTO) allocated by the European Southern Observatory (ESO) to the VIRMOS consortium, under a contractual agreement between the Centre National de la Recherche Scientifique of France, heading a consortium of French and Italian institutes, and ESO, to design, manufacture and test the VIMOS instrument. A. Pollo also acknowledges financial support from the Polish Ministry of Science, grant PBZ/MNiSW/07/2006/34A. Based on observations obtained with MegaPrime/MegaCam, a joint project of CFHT and CEA/DAPNIA, at the Canada-France-Hawaii Telescope (CFHT) which is operated by the National Research Council (NRC) of Canada, the Institut National des Science de l'Univers of the Centre National de la Recherche Scientifique (CNRS) of France, and the University of Hawaii. This work is based in part on data products produced at TERAPIX and the Canadian Astronomy Data Centre as part of the Canada-France-Hawaii Telescope Legacy Survey, a collaborative project of NRC and CNRS. The Millennium Simulation databases used in this paper and the web application providing online access to them were constructed as part of the activities of the German Astrophysical Virtual Observatory. 


\section{References}

Abell, G. O. 1958, ApJS, 3, 211

Adami, C., Mazure, A., Ilbert, O., et al. 2005, A\&A, 443, 805

Allen, S. W., Schmidt, R. W., \& Fabian, A. C. 2002, MNRAS, 334, L11

Andreon, S., Lobo, C., \& Iovino, A. 2004a, MNRAS, 349, 889

Andreon, S., Willis, J., Quintana, H., et al. 2004b, MNRAS, 353, 353

Andreon, S., Valtchanov, I., Jones, L. R., et al. 2005, MNRAS, 359, 1250

Andreon, S., Quintana, H., Tajer, M., Galaz, G., \& Surdej, J. 2006, MNRAS, 365,915

Andreon, S., Maughan, B., Trinchieri, G., \& Kurk, J. 2009, A\&A, 507, 147

Bahcall, N. A. 1981, ApJ, 247, 787

Barber, C. B., Dobkin, D. P., \& Huhdanpaa, H. 1996, ACM Transactions on Mathematical Software, 22, 469

Beers, T. C., Flynn, K., \& Gebhardt, K. 1990, AJ, 100, 32

Berlind, A. A., Frieman, J., Weinberg, D. H., et al. 2006, ApJS, 167, 1

Binney, J., \& Tremaine, S. 1988, Galactic Dynamics (Princeton Univiversity Press)

Blaizot, J., Wadadekar, Y., Guiderdoni, B., et al. 2005, MNRAS, 360, 159

Blanton, M. R. 2006, ApJ, 648, 268

Blanton, M. R., Eisenstein, D., Hogg, D. W., \& Zehavi, I. 2006, ApJ, 645, 977

Borgani, S., Gardini, A., Girardi, M., \& Gottlober, S. 1997, New Astron., 2, 119

Borgani, S., Girardi, M., Carlberg, R. G., Yee, H. K. C., \& Ellingson, E. 1999, ApJ, 527, 561

Bremer, M. N., Valtchanov, I., Willis, J., et al. 2006, MNRAS, 371, 1427

Bruzual, A. G., \& Charlot, S. 1993, ApJ, 405, 538

Butcher, H., \& Oemler, Jr., A. 1978, ApJ, 226, 559

Butcher, H., \& Oemler, A. 1984, ApJ, 285, 426

Carlberg, R. G., Yee, H. K. C., Morris, S. L., et al. 2001, ApJ, 552, 427

Coleman, G. D., Wu, C., \& Weedman, D. W. 1980, ApJS, 43, 393

Cooper, M. C., Newman, J. A., \& Coil, A. L. 2007, MNRAS, 376, 1445

Cucciati, O., Iovino, A., Marinoni, C., et al. 2006, A\&A, 458, 39

Davis, M., Faber, S. M., Newman, J., et al. 2003, in Discoveries and Research Prospects from 6- to 10-Meter-Class Telescopes II, Guhathakurta, Puragra, ed. P. Guhathakurta, Proc. SPIE, 4834, 161

De Lucia, G., \& Blaizot, J. 2007, MNRAS, 375, 2

De Propris, R., Stanford, S. A., Eisenhardt, P. R., \& Dickinson, M. 2003, ApJ, 598,20

De Propris, R., Colless, M., Peacock, J. A., et al. 2004, MNRAS, 351, 125

Delaunay, B. 1934, Bull. Acad. Sci. USSR, 7, 793

Donahue, M., Scharf, C. A., Mack, J., et al. 2002, ApJ, 569, 689

Dressler, A. 1980, ApJ, 236, 351

Dressler, A., Oemler, A. J., Couch, W. J., et al. 1997, ApJ, 490, 577

Eke, V. R., Baugh, C. M., Cole, S., et al. 2004, MNRAS, 348, 866

Ettori, S., Tozzi, P., \& Rosati, P. 2003, A\&A, 398, 879

Ettori, S., Morandi, A., Tozzi, P., et al. 2009, A\&A, 501, 61

Finoguenov, A., Watson, M. G., Tanaka, M., et al. 2010, MNRAS, 403, 2063

Franzetti, P., Scodeggio, M., Garilli, B., et al. 2007, A\&A, 465, 711

Garilli, B., Maccagni, D., \& Andreon, S. 1999, A\&A, 342, 408

Gavazzi, R., \& Soucail, G. 2007, A\&A, 462, 459

Gavazzi, R., Adami, C., Durret, F., et al. 2009, A\&A, 498, L33

Gehrels, N. 1986, ApJ, 303, 336

Gerke, B. F., Newman, J. A., Davis, M., et al. 2005, ApJ, 625, 6

Gerke, B. F., Newman, J. A., Faber, S. M., et al. 2007, MNRAS, 376, 1425

Gilbank, D. G., Bower, R. G., Castander, F. J., \& Ziegler, B. L. 2004, MNRAS, 348,551

Girardi, M., Biviano, A., Giuricin, G., Mardirossian, F., \& Mezzetti, M. 1993, ApJ, 404,38

Girardi, M., Borgani, S., Giuricin, G., Mardirossian, F., \& Mezzetti, M. 2000, ApJ, 530, 62

Giuricin, G., Samurović, S., Girardi, M., Mezzetti, M., \& Marinoni, C. 2001, ApJ, 554, 857

Gladders, M. D., \& Yee, H. K. C. 2000, AJ, 120, 2148

Goto, T. 2005, MNRAS, 356, L6

Goto, T., Okamura, S., Yagi, M., et al. 2003, PASJ, 55, 739

Hansen, S. M., McKay, T. A., Wechsler, R. H., et al. 2005, ApJ, 633, 122

Huchra, J. P., \& Geller, M. J. 1982, ApJ, 257, 423

Ilbert, O., Tresse, L., Zucca, E., et al. 2005, A\&A, 439, 863

Ilbert, O., Arnouts, S., McCracken, H. J., et al. 2006, A\&A, 457, 841

Iovino, A., McCracken, H. J., Garilli, B., et al. 2005, A\&A, 442, 423

Iovino, A., Cucciati, O., Scodeggio, M., et al. 2010, A\&A, 509, A40

Kauffmann, G., White, S. D. M., Heckman, T. M., et al. 2004, MNRAS, 353, 713

Kepner, J., Fan, X., Bahcall, N., et al. 1999, ApJ, 517, 78

Kneib, J., Hudelot, P., Ellis, R. S., et al. 2003, ApJ, 598, 804

Knobel, C., Lilly, S. J., Iovino, A., et al. 2009, ApJ, 697, 1842

Koester, B. P., McKay, T. A., Annis, J., et al. 2007, ApJ, 660, 221

Kovac, K., Lilly, S. J., Knobel, C., et al. 2010, ApJ, 718, 86
Le Fèvre, O., Mellier, Y., McCracken, H. J., et al. 2004, A\&A, 417, 839 Le Fèvre, O., Vettolani, G., Garilli, B., et al. 2005, A\&A, 439, 845 Ledlow, M. J., Voges, W., Owen, F. N., \& Burns, J. O. 2003, AJ, 126, 2740

Lemson, G., \& Virgo Consortium, T. 2006 [arXiv: astro-ph/0608019]

Lilly, S. J., Fèvre, O. L., Renzini, A., et al. 2007, ApJS, 172, 70

Lilly, S. J., LeBrun, V., Maier, C., et al. 2009, ApJS, 184, 218

Limousin, M., Cabanac, R., Gavazzi, R., et al. 2009, A\&A, 502, 445

Limousin, M., Ebeling, H., Ma, C., et al. 2010, MNRAS, 474

Margoniner, V. E., de Carvalho, R. R., Gal, R. R., \& Djorgovski, S. G. 2001, ApJ, 548, L143

Marinoni, C., \& Hudson, M. J. 2002, ApJ, 569, 101

Marinoni, C., Davis, M., Newman, J. A., \& Coil, A. L. 2002, ApJ, 580, 122

Materne, J. 1978, A\&A, 63, 401

Mazure, A., Adami, C., Pierre, M., et al. 2007, A\&A, 467, 49

McCracken, H. J., Radovich, M., Bertin, E., et al. 2003, A\&A, 410, 17

Meneux, B., Guzzo, L., Garilli, B., et al. 2008, A\&A, 478, 299

Miller, C. J., Nichol, R. C., Reichart, D., et al. 2005, AJ, 130, 968

Mirtich, B. 1996, Journal of graphics tools, 1, 31

Morandi, A., Pedersen, K., \& Limousin, M. 2010, ApJ, 713, 491

Newman, J. A., \& Davis, M. 2002, ApJ, 564, 567

Oemler, A. J. 1974, ApJ, 194, 1

Olsen, L. F., Benoist, C., Cappi, A., et al. 2007, A\&A, 461, 81

Pierre, M., Pacaud, F., Duc, P.-A., et al. 2006, MNRAS, 372, 591

Poggianti, B. M., von der Linden, A., De Lucia, G., et al. 2006, ApJ, 642, 188

Pollo, A., Meneux, B., Guzzo, L., et al. 2005, A\&A, 439, 887

Popesso, P., Böhringer, H., Brinkmann, J., Voges, W., \& York, D. G. 2004, A\&A, 423, 449

Popesso, P., Biviano, A., Böhringer, H., Romaniello, M., \& Voges, W. 2005, A\&A, 433, 431

Popesso, P., Biviano, A., Böhringer, H., \& Romaniello, M. 2007a, A\&A, 461, 397

Popesso, P., Biviano, A., Romaniello, M., \& Böhringer, H. 2007b, A\&A, 461, 411

Postman, M., \& Geller, M. J. 1984, ApJ, 281, 95

Radovich, M., Arnaboldi, M., Ripepi, V., et al. 2004, A\&A, 417, 51

Richard, J., Smith, G. P., Kneib, J., et al. 2010, MNRAS, 313

Rosati, P., Borgani, S., \& Norman, C. 2002, ARA\&A, 40, 539

Sheldon, E. S., Johnston, D. E., Masjedi, M., et al. 2009, ApJ, 703, 2232

Springel, V., White, S. D. M., Jenkins, A., et al. 2005, Nature, 435, 629

Sunyaev, R. A., \& Zeldovich, Y. B. 1972, Comm. Astrophys. Space Phys., 4, 173

Sunyaev, R. A., \& Zeldovich, I. B. 1980, ARA\&A, 18, 537

Temporin, S., Iovino, A., Bolzonella, M., et al. 2008, A\&A, 482, 81

Treu, T., Ellis, R. S., Kneib, J.-P., et al. 2003, ApJ, 591, 53

Tully, R. B. 1980, ApJ, 237, 390

Valtchanov, I., Pierre, M., Willis, J., et al. 2004, A\&A, 423, 75

Voronoi, G. F. 1908, J. Reine Angew. Math., 134, 198

Weinmann, S. M., van den Bosch, F. C., Yang, X., \& Mo, H. J. 2006, MNRAS, 366,2

Willis, J. P., Pacaud, F., Valtchanov, I., et al. 2005a, MNRAS, 363, 675

Willis, J. P., Pacaud, F., Valtchanov, I., et al. 2005b, MNRAS, 364, 751

Zhang, Y., Böhringer, H., Finoguenov, A., et al. 2006, A\&A, 456, 55

Zucca, E., Ilbert, O., Bardelli, S., et al. 2006, A\&A, 455, 879

Zwicky, F., Herzog, E., \& Wild, P. 1968, Catalogue of galaxies and of clusters of galaxies (Pasadena: California Institute of Technology (CIT), 1961-1968)

1 INAF-Osservatorio Astronomico di Brera, via Brera 28, 20021 Milan, Italy

e-mail: cucciati@brera.mi .astro.it

2 Laboratoire d'Astrophysique de Marseille, Université de Provence, CNRS, 38 rue Frederic Joliot-Curie, 13388 Marseille Cedex 13, France

3 Centre de Physique Théorique, UMR 6207, CNRS-Université de Provence, 13288 Marseille, France

4 INAF-Osservatorio Astronomico di Bologna, via Ranzani 1, 40127 Bologna, Italy

5 IASF-INAF, via Bassini 15, 20133 Milano, Italy

${ }^{6}$ Institute of Astro- and Particle Physics, Leopold-FranzensUniversity Innsbruck, Technikerstraße 25, 6020 Innsbruck, Austria

7 INAF - Osservatorio Astronomico di Trieste, via Tiepolo 11, 34143 Trieste, Italy

8 Université de Lyon, 69003 Lyon, France; Université Lyon 1, Observatoire de Lyon, 9 avenue Charles André, Saint-Genis Laval, 69230, France; CNRS, UMR 5574, Centre de Recherche 
Astrophysique de Lyon; École Normale Supérieure de Lyon, 69007 Lyon, France

9 Max Planck Institut für Extraterrestrische Physik (MPE), Giessenbachstrasse 1, 85748 Garching bei München, Germany

10 Universitätssternwarte München, Scheinerstrasse 1, 81679 München, Germany

11 IRA-INAF, via Gobetti 101, 40129 Bologna, Italy

12 Canada France Hawaii Telescope corporation, Mamalahoa Hwy, Kamuela, 96743, USA

13 Max Planck Institut für Astrophysik, 85741 Garching, Germany

14 Institut d'Astrophysique de Paris, UMR 7095, 98 bis Bvd Arago, 75014 Paris, France

15 Laboratoire d'Astrophysique de Toulouse-Tarbes, Université de Toulouse, CNRS, 14 Av. E. Belin, 31400 Toulouse, France

16 School of Physics \& Astronomy, University of Nottingham, University Park, Nottingham, NG72RD, UK
17 Astrophysical Institute Potsdam, An der Sternwarte 16, 14482 Potsdam, Germany

18 Observatoire de Paris, LERMA, 61 Avenue de l'Observatoire, 75014 Paris, France

19 Università di Bologna, Dipartimento di Astronomia, via Ranzani 1, 40127 Bologna, Italy

20 Integral Science Data Centre, ch. d’Écogia 16, 1290 Versoix, Switzerland

${ }^{21}$ Geneva Observatory, ch. des Maillettes 51, 1290 Sauverny, Switzerland

22 The Andrzej Soltan Institute for Nuclear Studies, ul. Hoza 69, 00-681 Warszawa, Poland

23 Astronomical Observatory of the Jagiellonian University, ul Orla 171, 30-244 Kraków, Poland

${ }^{24}$ Instituto de Astrofísica de Andalucía - CSIC. Apdo. de correos 3004, 18080 Granada, Spain 